\title{
Slightly Compressible Forchheimer Flows in Rotating Porous Media
}

\author{
Emine Celik ${ }^{a}$, Luan Hoang ${ }^{b}$, and Thinh $\mathrm{Kieu}^{c}$ \\ June 2, 2021 \\ ${ }^{a}$ Department of Mathematics, Sakarya University \\ 54050, Sakarya, Turkey \\ ${ }^{b}$ Department of Mathematics and Statistics, Texas Tech University \\ Box 41042, Lubbock, TX 79409-1042, U. S. A. \\ ${ }^{c}$ Department of Mathematics, University of North Georgia, Gainesville Campus \\ 3820 Mundy Mill Rd., Oakwood, GA 30566, U. S. A. \\ Email addresses: eminecelik@sakarya.edu.tr, luan.hoang@ttu.edu, \\ thinh.kieu@ung.edu
}

\begin{abstract}
We formulate the the generalized Forchheimer equations for the three-dimensional fluid flows in rotating porous media. By implicitly solving the momentum in terms of the pressure's gradient, we derive a degenerate parabolic equation for the density in the case of slightly compressible fluids and study its corresponding initial, boundary value problem. We investigate the nonlinear structure of the parabolic equation. The maximum principle is proved and used to obtain the maximum estimates for the solution. Various estimates are established for the solution's gradient, in the Lebesgue norms of any order, in terms of the initial and boundary data. All estimates contain explicit dependence on key physical parameters including the angular speed.
\end{abstract}

Keywords: porous media, compressible fluids, non-Darcy, Forchheimer, rotating fluids, a priori estimates, maximum principle, gradient estimates.

2020 Mathematics Subject Classification: 76S05, 76U60, 86A05, 35K20, 35K65.

\section{Contents}

1 Introduction and formulation of the problem

2 Preliminaries 7

2.1 Notation and elementary inequalities . . . . . . . . . . . 7 G

2.2 Properties of the function $X \ldots \ldots \ldots \ldots$

3 Maximum estimates 13 
4 Preparations for the gradient estimates 15

5 Gradient estimates (I) 21

6 Gradient estimates (II) 26

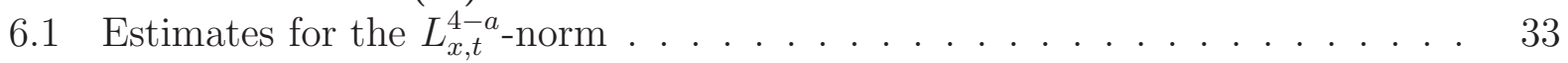

6.2 Estimates for higher $L_{x, t}^{s}$-norms $\ldots \ldots \ldots \ldots \ldots \ldots \ldots \ldots \ldots$

7 Gradient estimates (III) 43

\section{Introduction and formulation of the problem}

We study slightly compressible fluid flows in rotating porous media in the three dimensional space. The fluid has density $\rho$, velocity $v$, pressure $p$, and dynamic viscosity $\mu$. The porous medium has constant porosity $\tilde{\phi} \in(0,1)$ and permeability $k$. It is rotated with a constant angular velocity $\vec{\Omega}$, and an associated rotating frame is given. In this rotating coordinate system, $\vec{\Omega}$ is written as $\tilde{\Omega} \vec{k}$, where $\tilde{\Omega} \geq 0$ is the constant angular speed, and $\vec{k}$ is a constant unit vector. Let $x$ be the coordinate vector of a position in this rotating frame.

The equation for fluid flows written in the rotating frame, see e.g. [33], is

$$
\frac{\mu}{k} v+\frac{2 \rho \tilde{\Omega}}{\tilde{\phi}} \vec{k} \times v+\rho \tilde{\Omega}^{2} \vec{k} \times(\vec{k} \times x)=-\nabla p+\rho \vec{g},
$$

where $(2 \rho \tilde{\Omega} / \tilde{\phi}) \vec{k} \times v$ represents the Coriolis effect in porous media, $\tilde{\Omega}^{2} \vec{k} \times(\vec{k} \times x)$ is the centripetal acceleration, and $\vec{g}$ is the gravitational acceleration.

The basic assumption for equation (1.1) is that the flows obey the Darcy's law

$$
\frac{\mu}{k} v=-\nabla p+\rho \vec{g} .
$$

However, in many situations, for instance, when the Reynolds number is large, this assumption is invalid. Instead, Forchheimer equations [15,16] are usually used to model the flows in these cases. For example, the two-term Forchheimer's law states that

$$
a v+b|v| v=-\nabla+\rho \vec{g},
$$

where $a, b>0$ are some physical parameters. (See also Forchheimer's three-term and power laws in, e.g., [3, 28, 29].)

A general form of the Forchheimer equations, which extends (1.2) and (1.3) taking into account Muskat's dimension analysis [28], is

$$
\sum_{i=0}^{N} a_{i} \rho^{\alpha_{i}}|v|^{\alpha_{i}} v=-\nabla p+\rho \vec{g} .
$$

Here we focus on the explicit dependence on the density, leaving the dependence on the dynamic viscosity and permeability encoded in the coefficients $a_{i}$ 's. 
The interested reader is referred to the books [3, 29, 32] for more information about the Forchheimer equations and a larger family of Brinkman-Darcy-Forchheimer equations. For their mathematical analysis in the case of incompressible fluids, see e.g. [4, 5, 11, 18, 27, 30, 31] and references therein. For the treatments of compressible fluids, see [2,6, 7, 9, 10, 19, 24]. It is noted that the Forchheimer flows have drawn much less attention of mathematical research compared to the Darcy flows, and among the papers devoted to them, the number of those on compressible fluids is much smaller than that on the incompressible one.

Then the equation for the rotating flows corresponding to (1.4), written in the rotating frame, is

$$
\sum_{i=0}^{N} a_{i} \rho^{\alpha_{i}}|v|^{\alpha_{i}} v+\frac{2 \rho \tilde{\Omega}}{\tilde{\phi}} \vec{k} \times v+\rho \tilde{\Omega}^{2} \vec{k} \times(\vec{k} \times x)=-\nabla p+\rho \vec{g} .
$$

In particular, when $N=1$, the specific Forchheimer's two-term law for rotating fluids $[33,34$ is

$$
\frac{\mu}{k} v+\frac{c_{F} \rho}{\sqrt{k}}|v| v+\frac{2 \rho \tilde{\Omega}}{\tilde{\phi}} \vec{k} \times v+\rho \tilde{\Omega}^{2} \vec{k} \times(\vec{k} \times x)=-\nabla p+\rho \vec{g},
$$

where $c_{F}$ is the Forchheimer constant. Even in this case, there is no mathematical analysis for compressible fluids in literature.

We make one simplification in (1.5) $)$ replacing $\frac{2 \rho \tilde{\Omega}}{\tilde{\phi}} \vec{k} \times v$ with

$$
\frac{2 \rho_{*} \tilde{\Omega}}{\tilde{\phi}} \vec{k} \times v, \quad \rho_{*}=\text { const. } \geq 0 .
$$

We then approximate equation (1.5) by

$$
\sum_{i=0}^{N} a_{i} \rho^{\alpha_{i}}|v|^{\alpha_{i}} v+\mathcal{R} \vec{k} \times v+\rho \tilde{\Omega}^{2} \vec{k} \times(\vec{k} \times x)=-\nabla p+\rho \vec{g},
$$

where

$$
\mathcal{R}=\frac{2 \rho_{*} \tilde{\Omega}}{\tilde{\phi}}=\text { const. } \geq 0 .
$$

Let $g: \mathbb{R}^{+} \rightarrow \mathbb{R}^{+}$be a generalized polynomial defined by

$$
g(s)=a_{0}+a_{1} s^{\alpha_{1}}+\cdots+a_{N} s^{\alpha_{N}}=\sum_{i=0}^{N} a_{i} s^{\alpha_{i}} \quad \text { for } s \geq 0,
$$

where $N \geq 1$ is an integer, the powers $\alpha_{0}=0<\alpha_{1}<\alpha_{2}<\ldots<\alpha_{N}$ are real numbers, and the coefficients $a_{0}, a_{1}, \ldots, a_{N}$ are positive constants.

Then equation (1.7) can be rewritten as

$$
g(\rho|v|) v+\mathcal{R} \vec{k} \times v=-\nabla p+\rho \vec{g}-\rho \tilde{\Omega}^{2} \vec{k} \times(\vec{k} \times x) .
$$

Multiplying both sides of (1.10) by $\rho$ gives

$$
g(|\rho v|) \rho v+\mathcal{R} \vec{k} \times(\rho v)=-\rho \nabla p+\rho^{2} \vec{g}-\rho^{2} \tilde{\Omega}^{2} \vec{k} \times(\vec{k} \times x) .
$$

We will solve for $\rho v$ from (1.11), which is possible thanks to the following lemma. 
Lemma 1.1. Given any vector $k \in \mathbb{R}^{3}$, the function $F_{0}(v) \stackrel{\text { def }}{=} g(|v|) v+k \times v$ is a bijection from $\mathbb{R}^{3}$ to $\mathbb{R}^{3}$.

Proof. Note that $F_{0}$ is a continuous function on $\mathbb{R}^{3}$ and

$$
\frac{F_{0}(v) \cdot v}{|v|}=\frac{g(|v|)|v|^{2}}{|v|} \rightarrow \infty \text { as }|v| \rightarrow \infty .
$$

Then it is well-known that $F_{0}\left(\mathbb{R}^{3}\right)=\mathbb{R}^{3}$, see e.g. [13, Theorem 3.3].

It remains to prove that $F_{0}$ is one-to-one. Let $v, w \in \mathbb{R}^{3}$. We have

$$
\begin{aligned}
\left(F_{0}(v)-F_{0}(w)\right) \cdot(v-w) & =(g(|v|) v-g(|w|)) \cdot(v-w)+[k \times(v-w)] \cdot(v-w) \\
& =(g(|v|) v-g(|w|) w) \cdot(v-w) \\
& =a_{0}|v-w|^{2}+\sum_{i=1}^{N} a_{i}\left(|v|^{\alpha_{i}} v-|w|^{\alpha_{i}} w\right) \cdot(v-w) .
\end{aligned}
$$

By applying [14, Lemma 4.4, p. 13] to each $\left(|v|^{\alpha_{i}} v-|w|^{\alpha_{i}} w\right) \cdot(v-w)$, for $i \geq 1$, we then obtain

$$
\left(F_{0}(v)-F_{0}(w)\right) \cdot(v-w) \geq a_{0}|v-w|^{2}+\sum_{i=1}^{N} C_{i} a_{i}|v-w|^{\alpha_{i}+2},
$$

where $C_{i}>0$ depends on $\alpha_{i}$ and $a_{i}$, for $i=1, \ldots, N$.

If $F_{0}(v)=F_{0}(w)$, it follows the monotonicity (1.12) that

$$
0=\left(F_{0}(v)-F_{0}(w)\right) \cdot(v-w) \geq a_{0}|v-w|^{2},
$$

which implies $v=w$.

Let vector $\vec{k}=\left(k_{1}, k_{2}, k_{3}\right)$ be fixed now with $k_{1}^{2}+k_{2}^{2}+k_{3}^{2}=1$. We denote by $\mathbf{J}$ the $3 \times 3$ matrix for which $\mathbf{J} x=\vec{k} \times x$ for all $x \in \mathbb{R}^{3}$. Explicitly, we have

$$
\mathbf{J}=\left(\begin{array}{ccc}
0 & -k_{3} & k_{2} \\
k_{3} & 0 & -k_{1} \\
-k_{2} & k_{1} & 0
\end{array}\right) \quad \text { and } \mathbf{J}^{2}=\left(\begin{array}{ccc}
k_{1}^{2}-1 & k_{1} k_{2} & k_{1} k_{3} \\
k_{1} k_{2} & k_{2}^{2}-1 & k_{2} k_{3} \\
k_{1} k_{3} & k_{2} k_{3} & k_{3}^{2}-1
\end{array}\right)
$$

Definition 1.2. Throughout the paper, the function $g$ in (1.9) is fixed. We define the function $F: \mathbb{R}^{3} \rightarrow \mathbb{R}^{3}$ by

$$
F(v)=g(|v|) v+\mathcal{R} \mathbf{J} v \quad \text { for } v \in \mathbb{R}^{3},
$$

and denote its inverse function, which exists thanks to Lemma 1.1, by

$$
X=F^{-1} \text {. }
$$

Since $F$ is odd, then so is $X$. Returning to equation (1.11), we can invert

$$
\rho v=-X\left(\rho \nabla p-\rho^{2} \vec{g}+\rho^{2} \tilde{\Omega}^{2} \mathbf{J}^{2} x\right) .
$$


We recall that the fluid's compressibility for isothermal conditions is

$$
\kappa=-\frac{1}{V} \frac{d V}{d p}=\frac{1}{\rho} \frac{d \rho}{d p}
$$

where $V$, here, denotes the fluid's volume. In many cases such as (isothermal) compressible liquids, $\kappa$ is assumed to be a constant [3, 28]. In particular, it is a small positive constant for (isothermal) slightly compressible fluids such as crude oil and water. This condition is commonly used in petroleum and reservoir engineering [1,12], where the fluid dynamics in porous media have important applications. The current paper is focused on (isothermal) slightly compressible fluids, hence, we study the following equation of state

$$
\frac{1}{\rho} \frac{d \rho}{d p}=\kappa, \quad \text { where the constant compressibility } \kappa>0 \text { is small. }
$$

Using (1.17), we write (1.16) as

$$
\rho v=-X\left(\kappa^{-1} \nabla \rho-\rho^{2} \vec{g}+\rho^{2} \tilde{\Omega}^{2} \mathbf{J}^{2} x\right) .
$$

Consider the equation of continuity

$$
\tilde{\phi} \frac{\partial \rho}{\partial t}+\nabla \cdot(\rho v)=0
$$

where $t$ is the time variable. Combining (1.19) with (1.18) gives

$$
\tilde{\phi} \frac{\partial \rho}{\partial t}=\nabla \cdot\left(X\left(\kappa^{-1} \nabla \rho-\rho^{2} \vec{g}+\rho^{2} \tilde{\Omega}^{2} \mathbf{J}^{2} x\right)\right) .
$$

In the rotating frame, the gravitational field $\vec{g}$ becomes $\vec{g}(t)=\tilde{\mathcal{G}} e_{0}(t)$, with the gravitational constant $\tilde{\mathcal{G}}>0$ and smooth unit vector-valued function $e_{0}(t)$, for $t \in \mathbb{R}$.

We make a simple change of variable $u=\rho / \kappa$. Then we obtain from (1.20) the partial differential equation (PDE)

$$
\phi u_{t}=\nabla \cdot\left(X\left(\nabla u+u^{2} \mathcal{Z}(x, t)\right)\right)
$$

where

$$
\begin{gathered}
\mathcal{Z}(x, t)=-\mathcal{G} e_{0}(t)+\Omega^{2} \mathbf{J}^{2} x, \\
\phi=\kappa \tilde{\phi}, \quad \mathcal{G}=\kappa^{2} \tilde{\mathcal{G}}, \quad \Omega=\kappa \tilde{\Omega} .
\end{gathered}
$$

To reduce the complexity in our mathematical treatment, hereafter, we consider the involved parameters and all equations to be non-dimensional. This is allowed by using appropriate scalings.

In this paper, we study the initial and boundary value problem (IBVP) for equation (1.21). More specifically, let $U$ be an open, bounded set in $\mathbb{R}^{3}$ with $C^{1}$ boundary $\Gamma=\partial U$. We study the following problem

$$
\begin{cases}\phi u_{t}=\nabla \cdot\left(X\left(\nabla u+u^{2} \mathcal{Z}(x, t)\right)\right) & \text { in } U \times(0, \infty) \\ u(x, 0)=u_{0}(x) & \text { in } U \\ u(x, t)=\psi(x, t) & \text { in } \Gamma \times(0, \infty),\end{cases}
$$


where the initial data $u_{0}(x)$ and the Dirichlet boundary data $\psi(x, t)$ are given.

We will focus on the mathematical analysis of problem (1.23). We obtain various estimates of the solution in terms of the initial and boundary data. These estimates show how the solutions, in space and time, can be controlled by the initial and boundary data. We emphasize that the dependence on the problem's key parameters, including the angular speed of rotation, are expressed explicitly in our results.

The paper is organized as follows. In section 2, we establish basic properties of the function $X$ which are crucial to our understanding of problem (1.23). They reveal the nonlinear structure and the degeneracy of the nonlinear parabolic equation (1.21). Moreover, they have explicit dependence on the physical parameters, which, as stated above, is an important goal of this paper. In section 3, we prove the maximum principle for nonnegative solutions of equation (1.21) in Theorem 3.1. Using this, we derive the maximum estimates for non-negative solutions of the IBVP (1.23) in Corollary 3.2. Section 4 contains the Ladyženskaja-Ural'ceva-typed embedding, Theorem 4.2, with the weight $K[w, Q]$ which is related to the type of degeneracy of the nonlinear PDE (1.21). This is one of the key tools in obtaining higher integrability for the gradient later. In section 5, we establish the estimate for the $L_{x, t}^{2-a}$-norm of the gradient in Theorem 5.4. It was done through the $\mathcal{K}$-weighted $L^{2}$ norm first, see Proposition 5.1, and then by the interpretation of the weight $\mathcal{K}$. In section 6 , we estimate the $L_{x, t}^{s}$-norms of the gradient, which is interior in the spatial variables, for any finite number $s>2-a$. Specifically, we obtain estimates for $2-a<s \leq 4-a$ in subsection 6.1, and for $s>4-a$ in subsection 6.2. We use the iteration method by Ladyženskaja and

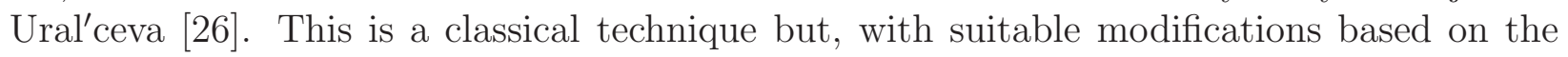
structure of equation (1.21), applies well to our complicated nonlinear PDE. Moreover, it is sufficiently explicit to allow us to track all the necessary constants. Section 7 is devoted to the estimates for the the gradient's $L_{t}^{\infty} L_{x}^{s}$-norms, which, of course, are stronger norms than those in the previous section.

It is worth mentioning that the derived estimates in this paper are already complicated, therefore, we strive to make them coherent, and hence more digestible, rather than sharp.

Concerning the simplification (1.6), it is a common strategy when encountering a new nonlinear problem. As presented above, it allows us to formulate the whole fluid dynamics system as a scalar parabolic equation (1.21). Such approximation, usually with some average density $\rho_{*}$, makes the problem much more accessible, while still gives insights on the flows' behaviors. More importantly, this approach prompts the way to analyze the full model. Indeed, in the general case, the $\mathcal{R}$ in (1.8) becomes $\mathcal{R}(u)$, and the PDE (1.21) becomes

$$
\phi u_{t}=\nabla \cdot\left(X\left(u, \nabla u+u^{2} \mathcal{Z}(x, t)\right)\right)
$$

with $X(u, y)$ defined in the same way as (1.15). Therefore, we can reduce the fluid dynamics system to a scalar PDE again. Furthermore, the properties of function $X$ established in subsection 2.2 with explicit dependence on $\mathcal{R}=\mathcal{R}(u)$, and other $X$-related results in section 4 will play fundamental roles in understating the structure of the PDE (1.24). This will be pursued and reported in a sequel of this paper.

Finally, we comment that the choice of the equation of state (1.17), in addition to its meaningful applications, yields the PDE (1.21) which can be analyzed rather thoroughly. Indeed, many strong estimates will be obtained in the next sections. In spite of this fo- 
cus on the slightly compressible fluids, the techniques developed in the current paper can be combined with those in our previous work [9, 10] to model and analyze other types of compressible fluid flows such as the rotating isentropic flows for gases.

\section{Preliminaries}

This section contains prerequisites and basic results on function $X$.

\subsection{Notation and elementary inequalities}

A vector $x \in \mathbb{R}^{3}$ is denoted by a 3 -tuple $\left(x_{1}, x_{2}, x_{3}\right)$ and considered as a column vector, i.e., a $3 \times 1$ matrix. Hence $x^{\mathrm{T}}$ is the $1 \times 3$ matrix $\left(x_{1} x_{2} x_{3}\right)$.

For a function $f=\left(f_{1}, f_{2}, \ldots, f_{m}\right): \mathbb{R}^{n} \rightarrow \mathbb{R}^{m}$, its derivative is the $m \times n$ matrix

$$
f^{\prime}=D f=\left(\frac{\partial f_{i}}{\partial x_{j}}\right)_{1 \leq i \leq m, 1 \leq j \leq n} .
$$

In particular, when $n=3$ and $m=1$, i.e., $f: \mathbb{R}^{3} \rightarrow \mathbb{R}$, the derivative is

$$
f^{\prime}=D f=\left(f_{x_{1}} f_{x_{2}} f_{x_{3}}\right),
$$

while its gradient vector is

$$
\nabla f=\left(f_{x_{1}}, f_{x_{2}}, f_{x_{3}}\right)=(D f)^{\mathrm{T}} .
$$

The Hessian matrix is

$$
D^{2} f \stackrel{\text { def }}{=} D(\nabla f)=\left(\frac{\partial^{2} f}{\partial x_{j} \partial x_{i}}\right)_{i, j=1,2,3} .
$$

Interpolation inequality for integrals:

$$
\int|f|^{s} d \mu \leq\left(\int|f|^{p} d \mu\right)^{\frac{q-s}{q-p}}\left(\int|f|^{q} d \mu\right)^{\frac{s-p}{q-p}} \text { for } 0<p<s<q .
$$

For two vectors $x, y \in \mathbb{R}^{3}$, their dot product is $x \cdot y=x^{\mathrm{T}} y=y^{\mathrm{T}} x$, while $x y^{\mathrm{T}}$ is the $3 \times 3$ matrix $\left(x_{i} y_{j}\right)_{i, j=1,2,3}$.

Let $\mathbf{A}=\left(a_{i j}\right)$ and $\mathbf{B}=\left(b_{i j}\right)$ be any $3 \times 3$ matrices of real numbers. Their inner product is

$$
\mathbf{A}: \mathbf{B} \stackrel{\text { def }}{=} \operatorname{trace}\left(\mathbf{A B}^{\mathrm{T}}\right)=\sum_{i, j=1}^{3} a_{i j} b_{i j} .
$$

The Euclidean norm of the matrix $\mathbf{A}$ is

$$
|\mathbf{A}|=(\mathbf{A}: \mathbf{A})^{1 / 2}=\left(\sum_{i, j=1}^{3} a_{i j}^{2}\right)^{1 / 2}
$$

(Note that we do not use $|\mathbf{A}|$ to denote the determinant in this paper.) 
When $\mathbf{A}$ is considered as a linear operator, another norm is defined by

$$
\|\mathbf{A}\|_{\text {op }}=\max \left\{\frac{|\mathbf{A} x|}{|x|}: x \in \mathbb{R}^{3}, x \neq 0\right\}=\max \left\{|\mathbf{A} x|: x \in \mathbb{R}^{3},|x|=1\right\} .
$$

We have the following inequalities

$$
\begin{aligned}
|\mathbf{A} y| & \leq|\mathbf{A}| \cdot|y| \quad \text { for any } y \in \mathbb{R}^{3}, \\
|\mathbf{A} y| & \leq\|\mathbf{A}\|_{\text {op }} \cdot|y| \quad \text { for any } y \in \mathbb{R}^{3}, \\
|\mathbf{A B}| & \leq|\mathbf{A}| \cdot|\mathbf{B}| .
\end{aligned}
$$

It is also known that

$$
\|\mathbf{A}\|_{\text {op }} \leq|\mathbf{A}| \leq c_{*}\|\mathbf{A}\|_{\text {op }}
$$

where $c_{*}$ is a positive constant independent of $\mathbf{A}$.

In particular, for matrix $\mathbf{J}$, we observe, for any $x \in \mathbb{R}^{3}$, that

$$
|\mathbf{J} x| \leq|\vec{k}||x|=|x| \quad \text { and } \quad\left|\mathbf{J}^{2} x\right| \leq|\mathbf{J} x| \leq|x|
$$

By choosing $x \neq 0$ perpendicular to $\vec{k}$, we conclude, for the norm (2.2), that

$$
\|\mathbf{J}\|_{\text {op }}=\left\|\mathbf{J}^{2}\right\|_{\text {op }}=1
$$

For the Euclidean norm, we have, from explicit formulas in (1.13), that

$$
\begin{gathered}
|\mathbf{J}|^{2}=2|\vec{k}|^{2}=2, \\
\left|\mathbf{J}^{2}\right|^{2}=3-2|\vec{k}|^{2}+|\vec{k}|^{4}=2 .
\end{gathered}
$$

We recall below some more elementary inequalities that will be used in this paper. First,

$$
\frac{x^{p}+y^{p}}{2} \leq(x+y)^{p} \leq 2^{(p-1)^{+}}\left(x^{p}+y^{p}\right) \text { for all } x, y \geq 0, p>0
$$

where $z^{+}=\max \{z, 0\}$ for any $z \in \mathbb{R}$. Particularly,

$$
(x+y)^{p} \leq 2^{p}\left(x^{p}+y^{p}\right) \text { for all } x, y \geq 0, p>0 .
$$

Second,

$$
\begin{gathered}
x^{\beta} \leq x^{\alpha}+x^{\gamma} \quad \text { for all } x, y \geq 0,0 \leq \alpha \leq \beta \leq \gamma \\
x^{\beta} \leq 1+x^{\gamma} \text { for all } x, y \geq 0,0 \leq \beta \leq \gamma .
\end{gathered}
$$

Above and throughout the paper, we conveniently use $0^{0}=1$.

By the triangle inequality and the second inequality of (2.10), we have

$$
|x-y|^{p} \geq 2^{-(p-1)^{+}}|x|^{p}-|y|^{p} \quad \text { for all } x, y \in \mathbb{R}^{n}, p>0 .
$$




\subsection{Properties of the function $X$}

It is obvious that the structure of the parabolic equation (1.21) depends greatly on the properties of the function $X$. Thus, this subsection is devoted to studying $X$.

Recall that the functions $F$ and $X$ are defined in Definition 1.2. Throughout the paper, we denote

$$
\begin{gathered}
a=\frac{\alpha_{N}}{1+\alpha_{N}} \in(0,1), \\
\chi_{0}=g(1)=\sum_{i=0}^{N} a_{i} \text { and } \chi_{1}=g(1)+\mathcal{R}=\chi_{0}+\mathcal{R} .
\end{gathered}
$$

Lemma 2.1. (i) One has

$$
\frac{c_{1} \chi_{1}^{-1}|y|}{(1+|y|)^{a}} \leq|X(y)| \leq \frac{c_{2} \chi_{1}^{a}|y|}{(1+|y|)^{a}} \text { for all } y \in \mathbb{R}^{3},
$$

where $c_{1}=\min \left\{1, \chi_{0}\right\}^{a}$ and $c_{2}=2^{a} c_{1}^{-1} \min \left\{a_{0}, a_{N}\right\}^{-1}$. Alternatively,

$$
\chi_{1}^{-(1-a)}|y|^{1-a}-1 \leq|X(y)| \leq c_{3}|y|^{1-a} \text { for all } y \in \mathbb{R}^{3},
$$

where $c_{3}=\left(a_{N}\right)^{a-1}$.

(ii) One has

$$
\frac{c_{4} \chi_{1}^{-2}|y|^{2}}{(1+|y|)^{a}} \leq X(y) \cdot y \leq \frac{c_{2} \chi_{1}^{a}|y|^{2}}{(1+|y|)^{a}} \text { for all } y \in \mathbb{R}^{3}
$$

where $c_{4}=\left(\min \left\{1, a_{0}, a_{N}\right\} / 2^{\alpha_{N}}\right)^{1+a}$. Alternatively,

$$
c_{5} \chi_{1}^{-2}\left(|y|^{2-a}-1\right) \leq X(y) \cdot y \leq c_{3}|y|^{2-a} \text { for all } y \in \mathbb{R}^{3},
$$

where $c_{5}=2^{-a} c_{4}$.

Proof. Let $y \in \mathbb{R}^{3}$ and $v=X(y)$. Then, by (1.15),

$$
F(v)=y \text {. }
$$

(i) Since $g(|v|) v$ and $\vec{k} \times v$ are orthogonal, we have from (1.14) and (2.21) that

$$
|y|^{2}=g(|v|)^{2}|v|^{2}+\mathcal{R}^{2}|\mathbf{J} v|^{2} .
$$

This and (2.7) show that

$$
g(|v|)^{2}|v|^{2} \leq|y|^{2} \leq\left(g(|v|)^{2}+\mathcal{R}^{2}\right)|v|^{2}
$$

Thus,

$$
g(|v|)|v| \leq|y| \leq(g(|v|)+\mathcal{R})|v| .
$$

Proof of (2.18). From the first inequality in (2.22),

$$
|y| \geq g(|v|)|v| \geq a_{N}|v|^{\alpha_{N}+1} \text {, which implies }|v| \leq\left(a_{N}^{-1}|y|\right)^{\frac{1}{\alpha_{N}+1}}=c_{3}|y|^{1-a} .
$$


So we obtain the second inequality of (2.18). From (2.22),

$$
|y| \leq \chi_{1}(1+|v|)^{\alpha_{N}+1} .
$$

Then we obtain the first inequality in (2.18).

Proof of (2.17). Since $X(0)=0$, we consider only $y, v \neq 0$.

Case $|v|>1$. By (2.22),$|y|>g(1) \cdot 1=\chi_{0}$. Furthermore, by (2.23),

$$
|v| \leq\left(a_{N}^{-1}|y|\right)^{1-a}=\frac{2^{a} a_{N}^{a-1}|y|}{(|y|+|y|)^{a}} \leq \frac{2^{a} \chi_{1}^{a} a_{N}^{-1}|y|}{\left(|y|+\chi_{0}\right)^{a}} \leq \frac{2^{a} a_{N}^{-1} \chi_{1}^{a}|y|}{\left(\min \left\{1, \chi_{0}\right\}\right)^{a}(|y|+1)^{a}} .
$$

On the other hand, we have from (2.22) that $|y| \leq\left(g(1)|v|^{\alpha_{N}}+\mathcal{R}\right)|v| \leq \chi_{1}|v|^{\alpha_{N}+1}$. Then

$$
|v| \geq\left(\chi_{1}^{-1}|y|\right)^{1-a} \geq \frac{\chi_{1}^{a-1}|y|}{|y|^{a}} \geq \frac{\chi_{0}^{a} \chi_{1}^{-1}|y|}{(|y|+1)^{a}} .
$$

Case $0<|v| \leq 1$. It follows (2.22) that $a_{0}|v| \leq|y| \leq \chi_{1}|v| \leq \chi_{1}$. Thus,

$$
\begin{aligned}
& |v| \leq a_{0}^{-1}|y|=\frac{a_{0}^{-1}|y|\left(|y|+\chi_{0}\right)^{a}}{\left(|y|+\chi_{0}\right)^{a}} \leq \frac{a_{0}^{-1}|y|\left(\chi_{1}+\chi_{0}\right)^{a}}{\left(|y|+\chi_{0}\right)^{a}} \leq \frac{a_{0}^{-1}\left(2 \chi_{1}\right)^{a}|y|}{\left(\min \left\{1, \chi_{0}\right\}\right)^{a}(|y|+1)^{a}} \\
& |v| \geq \chi_{1}^{-1}|y| \geq \chi_{1}^{-1}|y| \frac{1}{(|y|+1)^{a}}
\end{aligned}
$$

From (2.25) and (2.27), we obtain the second inequality in (2.17). From (2.26) and (2.28), we obtain the first inequality in (2.17).

(ii) Note that the second inequality of (2.19), respectively (2.20), follows the CauchySchwarz inequality and the second inequality of (2.17), respectively (2.18). Thus, we focus on proving the first inequalities of (2.19) and (2.20). We have from (1.14) and (2.22) that

$$
X(y) \cdot y=v \cdot F(v)=g(|v|)|v|^{2} \geq \frac{g(|v|)|y|^{2}}{(g(|v|)+\mathcal{R})^{2}}
$$

We estimate

$$
g(|v|)+\mathcal{R} \leq \chi_{1}(1+|v|)^{\alpha_{N}}
$$

and, by using (2.11),

$$
g(|v|) \geq \min \left\{a_{0}, a_{N}\right\}\left(1+|v|^{\alpha_{N}}\right) \geq \min \left\{a_{0}, a_{N}\right\} 2^{-\alpha_{N}}(1+|v|)^{\alpha_{N}} .
$$

Hence,

$$
\frac{g(|v|)}{(g(|v|)+\mathcal{R})^{2}} \geq \frac{\min \left\{a_{0}, a_{N}\right\} 2^{-\alpha_{N}}}{\chi_{1}^{2}(1+|v|)^{\alpha_{N}}}
$$

Note, by (2.10), that we also have

$$
1+|y| \geq 1+g(|v|)|v| \geq \min \left\{1, a_{N}\right\}\left(1+|v|^{\alpha_{N}+1}\right) \geq \min \left\{1, a_{N}\right\} 2^{-\alpha_{N}}(1+|v|)^{\alpha_{N}+1} .
$$


Then,

$$
(1+|v|)^{\alpha_{N}} \leq(1+|y|)^{a}\left(2^{\alpha_{N}} / \min \left\{1, a_{N}\right\}\right)^{a}
$$

Therefore,

$$
\frac{g(|v|)}{(g(|v|)+\mathcal{R})^{2}} \geq \frac{\min \left\{a_{0}, a_{N}\right\} 2^{-\alpha_{N}}}{\chi_{1}^{2}(1+|y|)^{a}}\left(\min \left\{1, a_{N}\right\} 2^{-\alpha_{N}}\right)^{a} \geq \frac{c_{4} \chi_{1}^{-2}}{(1+|y|)^{a}} .
$$

Hence we obtain the first inequality in (2.19). Then the first inequality of (2.20) follows this by considering $|y| \leq 1$ and $|y|>1$ separately.

Remark 2.2. Compared to (2.18), inequality in (2.17) indicates $X(y) \rightarrow 0$ as $|y| \rightarrow 0$ at the rate $|y|$, while $X(y) \rightarrow \infty$ as $|y| \rightarrow \infty$ at a different rate $|y|^{1-a}$. This refined form (2.17) and its proof originate from [8, Lemma 2.1].

Lemma 2.3. The function $F$ belongs to $C^{1}\left(\mathbb{R}^{3}\right)$, and the derivative matrix $F^{\prime}(v)$ is invertible for each $v \in \mathbb{R}^{3}$. Consequently, $X \in C^{1}\left(\mathbb{R}^{3}\right)$ and $X^{\prime}(y)$ is invertible for each $y \in \mathbb{R}^{3}$.

Proof. Elementary calculations show that

$$
\begin{aligned}
& F^{\prime}(v)=g^{\prime}(|v|) \frac{v v^{\mathrm{T}}}{|v|}+g(|v|) \mathbf{I}_{3}+\mathcal{R} \mathbf{J} \text { for } v \neq 0 \\
& F^{\prime}(0)=g(0) \mathbf{I}_{3}+\mathcal{R} \mathbf{J}
\end{aligned}
$$

Clearly, $F^{\prime}(v)$ is continuous at $v \neq 0$, and $F^{\prime}(v) \rightarrow F^{\prime}(0)$ as $v \rightarrow 0$. Therefore, $F \in C^{1}\left(\mathbb{R}^{3}\right)$.

For $z \in \mathbb{R}^{3}$, we have

$$
\begin{aligned}
& z^{\mathrm{T}} F^{\prime}(v) z=g^{\prime}(|v|) \frac{\left(z^{\mathrm{T}} v\right)^{2}}{|v|}+g(|v|)|z|^{2} \text { for } v \neq 0 \\
& z^{\mathrm{T}} F^{\prime}(0) z=g(0)|z|^{2}
\end{aligned}
$$

Since $g^{\prime}(s)>0$ for $s>0$, it follows that

$$
z^{\mathrm{T}} F^{\prime}(v) z \geq g(|v|)|z|^{2} \text { for all } v, z \in \mathbb{R}^{3} .
$$

Let $v \in \mathbb{R}^{3}$. If $F^{\prime}(v) z=0$, then $0=z^{\mathrm{T}} F^{\prime}(v) z \geq g(|v|)|z|^{2}$, which implies that $z=0$. Hence, $F^{\prime}(v)$ is invertible.

By the Inverse Function Theorem, the statements for $X$ follow those for $F$.

Lemma 2.4. For any $y \in \mathbb{R}^{3}$, the derivative matrix $X^{\prime}(y)$ satisfies

$$
\begin{gathered}
c_{6} \chi_{1}^{-1}(1+|y|)^{-a} \leq\left|X^{\prime}(y)\right| \leq c_{7}\left(1+\chi_{1}\right)^{a}(1+|y|)^{-a}, \\
\xi^{\mathrm{T}} X^{\prime}(y) \xi \geq c_{8} \chi_{1}^{-2}(1+|y|)^{-a}|\xi|^{2} \text { for all } \xi \in \mathbb{R}^{3},
\end{gathered}
$$

where

$$
c_{6}=\sqrt{3}\left(2^{-\alpha_{N}} \min \left\{1, a_{N}\right\}\right)^{a} /\left(\alpha_{N}+2\right), \quad c_{7}=c_{*} 2^{\alpha_{N}} / \min \left\{a_{0}, a_{N}\right\}, \quad c_{8}=c_{4} /\left(\alpha_{N}+2\right)^{2} .
$$


Proof. Let $y \in \mathbb{R}^{3}$, then, by (1.15), $X^{\prime}(y)=\left(F^{\prime}(v)\right)^{-1}$, with $F(v)=y$. We first claim that

$$
\frac{c_{9}}{g(|v|)+\mathcal{R}} \leq\left|X^{\prime}(y)\right| \leq \frac{c_{*}}{g(|v|)}
$$

where $c_{9}=\sqrt{3} /\left(\alpha_{N}+2\right)$, and $c_{*}>0$ is the positive constant in (2.6).

Accepting (2.38) for a moment, we prove the inequality (2.36). Observe, by (2.24), that

$$
1+|y| \leq\left(\chi_{1}+1\right)(1+|v|)^{\alpha_{N}} .
$$

On the one hand, (2.30) and (2.39) yield

$$
g(|v|) \geq \min \left\{a_{0}, a_{N}\right\} 2^{-\alpha_{N}}(1+|y|)^{a} /\left(1+\chi_{1}\right)^{a} .
$$

On the other hand, (2.29) and (2.31) give

$$
g(|v|)+\mathcal{R} \leq \chi_{1}(1+|y|)^{a}\left(2^{\alpha_{N}} / \min \left\{1, a_{N}\right\}\right)^{a} .
$$

Then, by combining (2.38), (2.40) and (2.41), we obtain (2.36).

We now prove the claim (2.38).

Proof of the first inequality in (2.38). First, we consider $y, v \neq 0$. In (2.33), the matrix $g^{\prime}(|v|)|v|^{-1} v v^{\mathrm{T}}+g(|v|) \mathbf{I}_{3}$ is symmetric, while $\mathcal{R} \mathbf{J}$ is anti-symmetric. Hence they are orthogonal, and, together with (2.8), we have

$$
\begin{aligned}
\left|F^{\prime}(v)\right|^{2} & =\left|g^{\prime}(|v|) \frac{v v^{\mathrm{T}}}{|v|}+g(|v|) \mathbf{I}_{3}\right|^{2}+\mathcal{R}^{2}|\mathbf{J}|^{2} \\
& =\operatorname{trace}\left\{\left(g^{\prime}(|v|) \frac{v v^{\mathrm{T}}}{|v|}+g(|v|) \mathbf{I}_{3}\right)^{2}\right\}+2 \mathcal{R}^{2} \\
& =\operatorname{trace}\left(\left(g^{\prime}(|v|)\right)^{2} v v^{\mathrm{T}}+2 g^{\prime}(|v|) g(|v|) \frac{v v^{\mathrm{T}}}{|v|}+(g(|v|))^{2} I_{3}\right)+2 \mathcal{R}^{2} .
\end{aligned}
$$

Since $\operatorname{trace}\left(v v^{\mathrm{T}}\right)=|v|^{2}$, we have

$$
\begin{aligned}
\left|F^{\prime}(v)\right|^{2} & =\left(g^{\prime}(|v|)\right)^{2}|v|^{2}+2 g^{\prime}(|v|) g(|v|)|v|+3(g(|v|))^{2}+2 \mathcal{R}^{2} \\
& =\left(g^{\prime}(|v|)|v|+g(|v|)\right)^{2}+2 g(|v|)^{2}+2 \mathcal{R}^{2}
\end{aligned}
$$

Note that

$$
g^{\prime}(|v|)>0 \quad \text { and } \quad g^{\prime}(|v|)|v| \leq \alpha_{N} g(|v|)
$$

Then

$$
\left|F^{\prime}(v)\right|^{2} \leq\left(\left(\alpha_{N}+1\right)^{2}+2\right) g(|v|)^{2}+2 \mathcal{R}^{2} \leq\left(\alpha_{N}+2\right)^{2}(g(|v|)+\mathcal{R})^{2} .
$$

Similarly, we have from (2.34) that

$$
\left|F^{\prime}(0)\right|^{2}=3 g(0)^{2}+2 \mathcal{R}^{2} \leq 3(g(0)+\mathcal{R})^{2} \leq\left(\alpha_{N}+2\right)^{2}(g(0)+\mathcal{R})^{2} .
$$

From (2.42) and (2.43), it follows

$$
\left|F^{\prime}(v)\right| \leq\left(\alpha_{N}+2\right)(g(|v|)+\mathcal{R}) \text { for all } v \in \mathbb{R}^{3} .
$$


By (2.5),

$$
\sqrt{3}=\left|\mathbf{I}_{3}\right|=\left|F^{\prime}(v) X^{\prime}(y)\right| \leq\left|F^{\prime}(v)\right| \cdot\left|X^{\prime}(y)\right|
$$

which gives

$$
\left|X^{\prime}(y)\right| \geq \frac{\sqrt{3}}{\left|F^{\prime}(v)\right|} \geq \frac{\sqrt{3}}{\left(\alpha_{N}+2\right)(g(|v|)+\mathcal{R})}=\frac{c_{9}}{g(|v|)+\mathcal{R}} .
$$

Proof of the second inequality in (2.38). For $z \neq 0$, by Cauchy-Schwarz inequality and (2.35), we have

$$
\left|F^{\prime}(v) z\right| \geq \frac{\left|z^{\mathrm{T}} F^{\prime}(v) z\right|}{|z|} \geq g(|v|)|z|
$$

For any $\xi \in \mathbb{R}^{3} \backslash\{0\}$, applying (2.45) to $z=X^{\prime}(y) \xi$, which is non-zero thanks to $X^{\prime}(y)$ being invertible, gives

$$
|\xi| \geq g(|v|)\left|X^{\prime}(y) \xi\right|
$$

Thus, the operator norm of $X^{\prime}(y)$ is bounded by $\left\|X^{\prime}(y)\right\|_{\text {op }} \leq 1 / g(|v|)$, and then, by relation (2.6),

$$
\left|X^{\prime}(y)\right| \leq c_{*}\left\|X^{\prime}(y)\right\|_{\text {op }} \leq c_{*} / g(|v|)
$$

Proof of (2.37). Let $u=X^{\prime}(y) \xi=\left(F^{\prime}(v)\right)^{-1} \xi$, which gives $\xi=F^{\prime}(v) u$. Rewriting $\left[X^{\prime}(y) \xi\right] \cdot \xi$ in terms of $u, v$ and using property (2.35), we have

$$
\left[X^{\prime}(y) \xi\right] \cdot \xi=u \cdot\left[F^{\prime}(v) u\right] \geq|u|^{2} g(|v|)=\left|X^{\prime}(y) \xi\right|^{2} g(|v|)
$$

From (2.3) and (2.44), for any $\xi \in \mathbb{R}^{3}$ :

$$
|\xi|=\left|F^{\prime}(v) X^{\prime}(y) \xi\right| \leq\left|F^{\prime}(v)\right| \cdot\left|X^{\prime}(y) \xi\right| \leq\left(\alpha_{N}+2\right)(g(|v|)+\mathcal{R})\left|X^{\prime}(y) \xi\right|,
$$

thus

$$
\left|X^{\prime}(y) \xi\right| \geq \frac{|\xi|}{\left(\alpha_{N}+2\right)(g(|v|)+\mathcal{R})} .
$$

Combining (2.46), (2.47) and (2.32) yields

$$
\xi^{\mathrm{T}} X^{\prime}(y) \xi \geq \frac{g(|v|)}{\left(\alpha_{N}+2\right)^{2}(g(|v|)+\mathcal{R})^{2}}|\xi|^{2} \geq \frac{c_{4} \chi_{1}^{-2}|\xi|^{2}}{\left(\alpha_{N}+2\right)^{2}(1+|y|)^{a}} .
$$

This proves (2.37).

\section{Maximum estimates}

We will estimate the solutions of (1.23) by the maximum principle. Denote

$$
\Phi(x, t)=\nabla u(x, t)+u^{2}(x, t) \mathcal{Z}(x, t)
$$


We re-write the PDE in (1.23) in the non-divergence form as

$$
\begin{aligned}
\phi u_{t}=X^{\prime}(\Phi):(D \Phi)^{\mathrm{T}} & =X^{\prime}(\Phi):\left(D^{2} u+u^{2} D \mathcal{Z}(x, t)+2 u \mathcal{Z}(x, t) D u\right)^{\mathrm{T}} \\
& =\mathbf{A}:\left(D^{2} u+u^{2} \Omega^{2} \mathbf{J}^{2}+2 u(\mathcal{Z}(x, t) D u)^{\mathrm{T}}\right),
\end{aligned}
$$

where $\mathbf{A}=\mathbf{A}(x, t)=X^{\prime}(\Phi(x, t))$.

We write $\mathbf{A}=\mathbf{S}+\mathbf{T}$, where $\mathbf{S}$ and $\mathbf{T}$ are the symmetric and anti-symmetric parts of $\mathbf{A}$, i.e.,

$$
\mathbf{S}=\left(s_{i j}\right)_{i, j=1,2,3}=\frac{1}{2}\left(\mathbf{A}+\mathbf{A}^{\mathrm{T}}\right) \quad \text { and } \quad \mathbf{T}=\frac{1}{2}\left(\mathbf{A}-\mathbf{A}^{\mathrm{T}}\right) .
$$

Sine $D^{2} u$ is symmetric, we have $\mathbf{T}: D^{2} u=0$, hence $\mathbf{A}: D^{2} u=\mathbf{S}: D^{2} u$.

Similarly, $\mathbf{J}^{2}$ is symmetric, and we have $\mathbf{A}: \mathbf{J}^{2}=\mathbf{S}: \mathbf{J}^{2}$.

Therefore,

$$
\phi u_{t}=\mathbf{S}: D^{2} u+u^{2} \Omega^{2} \mathbf{S}: \mathbf{J}^{2}+2 u \mathbf{A}:(\mathcal{Z}(x, t) D u)^{\mathrm{T}} .
$$

Equation (3.2) turns out to possess a maximum principle. Recall that the parabolic boundary of $U \times(0, T]$ is

$$
\partial_{p}(U \times(0, T])=\bar{U} \times[0, T] \backslash U \times(0, T] .
$$

Theorem 3.1 (Maximum principle). Suppose $T>0$, and $u \in C(\bar{U} \times[0, T]) \cap C_{x, t}^{2,1}(U \times(0, T])$ with $u \geq 0$ is a solution of (1.21) on $U \times(0, T]$. Then

$$
\max _{\bar{U} \times[0, T]} u=\max _{\partial_{p}(U \times(0, T])} u .
$$

Proof. We make use of equation (3.2) which is equivalent to (1.21). We examine the second term on the right-hand side of (3.2). Direct calculations using the formula of $\mathbf{J}^{2}$ in (1.13) give

$$
\mathbf{S}: \mathbf{J}^{2}=\sum_{i, j=1}^{3} k_{i} k_{j} s_{i j}-\sum_{i=1}^{3} s_{i i}=\vec{k}^{\mathrm{T}} \mathbf{S} \vec{k}-\operatorname{trace}(\mathbf{S}) .
$$

By (2.37), we have $\xi^{\mathrm{T}} \mathbf{A} \xi \geq 0$ for all $\xi \in \mathbb{R}^{3}$, and, hence,

$$
\xi^{\mathrm{T}} \mathbf{S} \xi \geq 0 \text { for all } \xi \in \mathbb{R}^{3} .
$$

By (3.4) and the fact $\mathbf{S}$ is symmetric, we have $\mathbf{S} \geq 0$ with eigenvalues $\lambda_{1}, \lambda_{2}, \lambda_{3} \geq 0$. Then, applying Cauchy-Schwarz's inequality and (2.4) to $\vec{k}^{\mathrm{T}} \mathbf{S} \vec{k}$, we obtain

$$
\mathbf{S}: \mathbf{J}^{2} \leq\|\mathbf{S}\|_{\text {op }}|\vec{k}|^{2}-\operatorname{trace}(\mathbf{S})=\max \left\{\lambda_{1}, \lambda_{2}, \lambda_{3}\right\} \cdot 1-\left(\lambda_{1}+\lambda_{2}+\lambda_{3}\right) \leq 0 .
$$

Let $\varepsilon>0$. Set $u^{\varepsilon}(x, t)=e^{-\varepsilon t} u(x, t)$ and $M_{\varepsilon}=\max _{\bar{U} \times[0, T]} u^{\varepsilon}$. We prove that

$$
\max _{\bar{U} \times[0, T]} u^{\varepsilon}=\max _{\partial_{p}(U \times(0, T])} u^{\varepsilon} .
$$

Suppose (3.6) is false. Then $M_{\varepsilon}>0$ and there exists a point $\left(x_{0}, t_{0}\right) \in U \times(0, T]$ for such that $u^{\varepsilon}\left(x_{0}, t_{0}\right)=M_{\varepsilon}$. At this maximum point $\left(x_{0}, t_{0}\right)$ we have

$$
u_{t}^{\varepsilon}\left(x_{0}, t_{0}\right) \geq 0, \quad \nabla u^{\varepsilon}\left(x_{0}, t_{0}\right)=0, \quad D^{2} u^{\varepsilon}\left(x_{0}, t_{0}\right) \leq 0 .
$$


We observe the followings:

(a) The second property of (3.7) implies $\nabla u\left(x_{0}, t_{0}\right)=0$.

(b) On the one hand, we have from (3.4) that $\mathbf{S}(x, t) \geq 0$ on $U \times(0, T]$. On the other hand, the last property of (3.7) implies $D^{2} u\left(x_{0}, t_{0}\right) \leq 0$. Then it is well-known that $\mathbf{S}\left(x_{0}, t_{0}\right)$ : $D^{2} u\left(x_{0}, t_{0}\right) \leq 0$, see e.g. [17, Chapter 2, Lemma 1].

From (3.2), (3.5), and (a), (b), we obtain $u_{t}\left(x_{0}, t_{0}\right) \leq 0$. Therefore,

$$
u_{t}^{\varepsilon}\left(x_{0}, t_{0}\right)=-\varepsilon e^{-\varepsilon t_{0}} u\left(x_{0}, t_{0}\right)+e^{-\varepsilon t_{0}} u_{t}\left(x_{0}, t_{0}\right) \leq-\varepsilon u^{\varepsilon}\left(x_{0}, t_{0}\right)=-\varepsilon M_{\varepsilon}<0 .
$$

This contradicts the first inequality in (3.7), hence, (3.6) holds true. Note that

$$
e^{-\varepsilon T} \max _{\bar{U} \times[0, T]} u \leq \max _{\bar{U} \times[0, T]} u^{\varepsilon}=\max _{\partial_{p}(U \times(0, T])} u^{\varepsilon} \leq \max _{\partial_{p}(U \times(0, T])} u \leq \max _{\bar{U} \times[0, T]} u .
$$

Then letting $\varepsilon \rightarrow 0$ yields (3.3).

Corollary 3.2. Let $u \in C(\bar{U} \times[0, T]) \cap C_{x, t}^{2,1}(U \times(0, T])$ with $u \geq 0$ solve problem (1.23) on a time interval $[0, T]$ for some $T>0$. Then it holds for all $t \in[0, T]$ that

$$
\max _{x \in \bar{U}} u(x, t) \leq M_{0}(t) \stackrel{\text { def }}{=} \sup _{x \in U}\left|u_{0}(x)\right|+\sup _{(x, \tau) \in \partial U \times(0, t]}|\psi(x, \tau)|
$$

Proof. Because of the continuity of $u$ on $\bar{U} \times[0, t]$, the quantity $M_{0}(t)$, in fact, is an upper bound of the maximum of $u$ on $\partial_{p}(U \times(0, t])$. Then applying inequality (3.3) to $T=t$ yields estimate (3.8).

\section{Preparations for the gradient estimates}

This section contains technical preparations for the estimates for different norms of the gradient $\nabla u$ in the next three sections.

Given two mappings $Q: U \rightarrow \mathbb{R}^{3}$ and $w: U \rightarrow \mathbb{R}$, we define a function $K[w, Q]$ on $U$ by

$$
K[w, Q](x)=\left(1+\left|\nabla w(x)+w^{2}(x) Q(x)\right|\right)^{-a} \quad \text { for } x \in U .
$$

This function will be conveniently used in comparisons with $X\left(\nabla w(x)+w^{2}(x) Q(x)\right)$ arising in the PDE (1.21).

Lemma 4.1. If $s \geq a$, then the following inequalities hold on $U$ :

$$
\begin{aligned}
& K[w, Q]|\nabla w|^{s} \leq 2^{2 s-a}|\nabla w|^{s-a}+2^{2 s+1-a}\left(1+\left|w^{2} Q\right|^{s}\right), \\
& K[w, Q]|\nabla w|^{s} \geq 3^{-1}|\nabla w|^{s-a}-3^{-1}\left(1+\left|w^{2} Q\right|^{s}\right) .
\end{aligned}
$$

Proof. We denote, in this proof, $K=K[w, Q]$. Let $s \geq a$, by the triangle inequality and inequalities (2.11), (2.13), we have

$$
\begin{aligned}
K|\nabla w|^{s} & \leq 2^{s} K \cdot\left(\left|\nabla w+w^{2} Q\right|^{s}+\left|w^{2} Q\right|^{s}\right) \\
& \leq 2^{s}\left|\nabla w+w^{2} Q\right|^{s-a}+2^{s} K\left|w^{2} Q\right|^{s} \\
& \leq 2^{s} \cdot 2^{s-a}\left(|\nabla w|^{s-a}+\left|w^{2} Q\right|^{s-a}\right)+2^{s} K\left|w^{2} Q\right|^{s} .
\end{aligned}
$$


Using inequality (2.13) and the fact $K \leq 1$, we estimate

$$
\left|w^{2} Q\right|^{s-a} \leq 1+\left|w^{2} Q\right|^{s}, \quad K\left|w^{2} Q\right|^{s} \leq 1+\left|w^{2} Q\right|^{s} .
$$

Hence,

$$
K|\nabla w|^{s} \leq 2^{2 s-a}|\nabla w|^{s-a}+\left(2^{2 s-a}+2^{s}\right)\left(1+\left|w^{2} Q\right|^{s}\right) .
$$

Noticing that $2^{2 s-a}+2^{s} \leq 2 \cdot 2^{2 s-a}$, we obtain (4.2) from (4.4).

To prove (4.3), we write $|\nabla w|^{s-a}=K|\nabla w|^{s-a}\left(1+\left|\nabla w+w^{2} Q\right|\right)^{a}$, and apply inequality (2.10) to have

$$
|\nabla w|^{s-a} \leq K|\nabla w|^{s-a}\left(1+|\nabla w|^{a}+\left|w^{2} Q\right|^{a}\right)=K \cdot\left(|\nabla w|^{s-a}+|\nabla w|^{s}+|\nabla w|^{s-a}\left|w^{2} Q\right|^{a}\right) .
$$

Concerning the last sum between the parentheses, applying inequality (2.13) to its first term gives

$$
|\nabla w|^{s-a} \leq 1+|\nabla w|^{s}
$$

and applying Young's inequality to its last term with the conjugate powers $s /(s-a)$ and $s / a$, when $s>a$, gives

$$
|\nabla w|^{s-a}\left|w^{2} Q\right|^{a} \leq|\nabla w|^{s}+\left|w^{2} Q\right|^{s} .
$$

Obviously, this inequality also holds when $s=a$. Thus,

$$
|\nabla w|^{s-a} \leq 3 K|\nabla w|^{s}+K \cdot\left(1+\left|w^{2} Q\right|^{s}\right) \leq 3 K|\nabla w|^{s}+\left(1+\left|w^{2} Q\right|^{s}\right) .
$$

We obtain (4.3).

For our later convenience, we rewrite inequality (4.5), by replacing $s-a$ with $s$, as

$$
|\nabla w|^{s} \leq 3 K[w, Q]|\nabla w|^{s+a}+\left(1+\left|w^{2} Q\right|^{s+a}\right) \text { for all } s \geq 0 .
$$

Theorem 4.2. For each $s \geq 1$, there exists a constant $C>0$ depending only on $s$ and number a in (2.15) such that for any function $w \in C^{2}(U)$ and non-negative function $\zeta \in C_{c}^{1}(U)$, the following inequality holds

$$
\begin{aligned}
\int_{U} K[w, Q]|\nabla w|^{2 s+2} \zeta^{2} d x \leq & C \sup _{\operatorname{supp} \zeta}\left(w^{2}\right)\left\{\int_{U} K[w, Q]\left|D^{2} w\right|^{2}\left(|\nabla w|^{2 s-2}+1\right) \zeta^{2} d x\right. \\
& \left.+\int_{U} K[w, Q]|\nabla w|^{2 s}\left(|\nabla \zeta|^{2}+\left(|w|\left|Q^{\prime}\right|+|Q|\right)^{2} \zeta^{2} w^{2}\right) d x\right\} \\
& +C \int_{U}\left(1+\left|w^{2} Q\right|^{2 s}\right)\left|w^{2} Q\right|^{2 s+2} \zeta^{2} d x
\end{aligned}
$$

Assume, in addition, that $Q$ and $Q^{\prime}$ are bounded on $U$. Then,

$$
\begin{aligned}
& \int_{U} K[w, Q]|\nabla w|^{2 s+2} \zeta^{2} d x \leq C M_{w}^{2} \int_{U} K[w, Q]\left|D^{2} w\right|^{2}|\nabla w|^{2 s-2} \zeta^{2} d x \\
& \quad+C M_{w}^{2} \int_{U} K[w, Q]|\nabla w|^{2 s}\left(|\nabla \zeta|^{2}+\left(M_{w} \mu_{Q}+M_{Q}\right)^{2} M_{w}^{2} \zeta^{2}\right) d x \\
& \quad+C \operatorname{sgn}(s-1) M_{w}^{2} \int_{U} K[w, Q]\left|D^{2} w\right|^{2} \zeta^{2} d x+C|U|\left(M_{w}^{2} M_{Q}\right)^{2 s+2}\left(1+M_{w}^{2} M_{Q}\right)^{2 s},
\end{aligned}
$$


where

$$
M_{w}=\sup _{x \in \operatorname{supp} \zeta}|w(x)|, \quad M_{Q}=\sup _{x \in U}|Q(x)|, \quad \mu_{Q}=\sup _{x \in U}\left|Q^{\prime}(x)\right| .
$$

Proof. For convenience in computing the derivatives, we will first establish (4.7) with $K[w, Q]$ being replaced by the following function

$$
K_{*}(x)=\left(1+\left|\nabla w(x)+w^{2}(x) Q(x)\right|^{2}\right)^{-a / 2} .
$$

In this proof, the symbol $C$ denotes a generic positive constant depending only on $s$ and number $a$ in (2.15), while $C_{\varepsilon}>0$ depends on $s, a$ and $\varepsilon$.

We use Einstein's summation convention in our calculations. Let

$$
I=\int_{U} K_{*}(x)|\nabla w(x)|^{2 s+2} \zeta^{2}(x) d x
$$

By integration by parts and direct calculations, we see that

$$
\begin{aligned}
I & =\int_{U}\left(K_{*}|\nabla w|^{2 s} \partial_{i} w \zeta^{2}\right) \cdot \partial_{i} w d x=-\int_{U} \partial_{i}\left(K_{*}|\nabla w|^{2 s} \partial_{i} w \zeta^{2}\right) \cdot w d x \\
& =I_{1}+I_{2}+I_{3}+I_{4}
\end{aligned}
$$

where

$$
\begin{aligned}
& I_{1}=a \int_{U} \frac{\left(\nabla w+w^{2} Q\right) \cdot\left(\partial_{i} \nabla w+2 w \partial_{i} w Q+w^{2} \partial_{i} Q\right)}{\left(1+\left|\nabla w+w^{2} Q\right|^{2}\right)^{a / 2+1}}|\nabla w|^{2 s} \partial_{i} w \cdot \zeta^{2} \cdot w d x, \\
& I_{2}=-\int_{U} K_{*}|\nabla w|^{2 s} \Delta w \cdot \zeta^{2} \cdot w d x \\
& I_{3}=-2 s \int_{U} K_{*} \cdot\left(|\nabla w|^{2 s-2} \partial_{i} \partial_{j} w \partial_{j} w\right) \cdot \partial_{i} w \cdot \zeta^{2} \cdot w d x \\
& I_{4}=-2 \int_{U} K_{*}|\nabla w|^{2 s} \partial_{i} w \zeta \partial_{i} \zeta \cdot w d x .
\end{aligned}
$$

Above, we used $\partial_{i}\left(|\nabla w|^{2 s}\right)=\partial_{i}\left(|\nabla w|^{2}\right)^{s}=s\left(|\nabla w|^{2}\right)^{s-1} \partial_{i}\left(|\nabla w|^{2}\right)$ to avoid possible singularities when $\nabla w=0$.

We estimate $I_{1}$ first. Observe that

$$
\frac{1}{\left(1+\left|\nabla w+w^{2} Q\right|^{2}\right)^{a / 2+1}}=\frac{K_{*}}{1+\left|\nabla w+w^{2} Q\right|^{2}} \leq \frac{2 K_{*}}{\left(1+\left|\nabla w+w^{2} Q\right|\right)^{2}} .
$$

By Cauchy-Schwarz and triangle inequalities, we have

$$
\begin{aligned}
I_{1} \leq & C \int_{U} \frac{K_{*}}{\left(1+\left|\nabla w+w^{2} Q\right|\right)^{2}} \cdot\left|\nabla w+w^{2} Q\right| \\
& \cdot\left(\left|D^{2} w\right||\nabla w|^{2 s+1}+|w||\nabla w|^{2 s+2}|Q|+w^{2}|\nabla w|^{2 s+1}\left|Q^{\prime}\right|\right) \zeta^{2} \cdot|w| d x \\
& \leq J_{1}+J_{2}+J_{3},
\end{aligned}
$$


where

$$
\begin{aligned}
& J_{1}=C \int_{U} \frac{K_{*}}{1+\left|\nabla w+w^{2} Q\right|}\left(\left|D^{2} w\right||\nabla w|^{2 s+1}\right) \zeta^{2} \cdot|w| d x, \\
& J_{2}=C \int_{U} \frac{K_{*}}{1+\left|\nabla w+w^{2} Q\right|}\left(|w Q||\nabla w|^{2 s+2}\right) \zeta^{2} \cdot|w| d x, \\
& J_{3}=C \int_{U} \frac{K_{*}}{1+\left|\nabla w+w^{2} Q\right|}\left(w^{2}\left|Q^{\prime}\right||\nabla w|^{2 s+1}\right) \zeta^{2} \cdot|w| d x .
\end{aligned}
$$

For $J_{1}$, by using triangle inequality $|\nabla w| \leq\left|\nabla w+w^{2} Q\right|+\left|w^{2} Q\right|$, we have

$$
\begin{aligned}
J_{1} & \leq C \int_{U} \frac{K_{*}}{1+\left|\nabla w+w^{2} Q\right|}\left(\left|\nabla w+w^{2} Q\right|^{2 s+1}+\left|w^{2} Q\right|^{2 s+1}\right)\left|D^{2} w\right| \zeta^{2}|w| d x \\
& \leq C \int_{U} K_{*} \cdot\left(\left|\nabla w+w^{2} Q\right|^{2 s}+\left|w^{2} Q\right|^{2 s+1}\right)\left|D^{2} w\right| \zeta^{2}|w| d x .
\end{aligned}
$$

Applying triangle inequality and (2.11) gives

$$
\left|\nabla w+w^{2} Q\right|^{2 s} \leq C\left(|\nabla w|^{2 s}+\left|w^{2} Q\right|^{2 s}\right)
$$

Thus,

$$
J_{1} \leq C \int_{U} K_{*}|\nabla w|^{2 s}\left|D^{2} w\right| \zeta^{2}|w| d x+C \int_{U} K_{*} \cdot\left(\left|w^{2} Q\right|^{2 s}+\left|w^{2} Q\right|^{2 s+1}\right)\left|D^{2} w\right| \zeta^{2}|w| d x .
$$

Let $\varepsilon>0$. Denote

$$
I_{5}=\int_{U} K_{*}\left|D^{2} w\right|^{2}|\nabla w|^{2 s-2} \zeta^{2} w^{2} d x, \quad I_{6}=\int_{U} K_{*}\left|D^{2} w\right|^{2} \zeta^{2} w^{2} d x
$$

In the last inequality for $J_{1}$, we apply Cauchy's inequality to obtain

$$
\begin{aligned}
J_{1} & \leq C \int_{U} K_{*}|\nabla w|^{2 s}\left|D^{2} w\right| \zeta^{2}|w| d x+C \int_{U} K_{*} \cdot\left(\left|w^{2} Q\right|^{2 s}+\left|w^{2} Q\right|^{2 s+1}\right)\left|D^{2} w\right| \zeta^{2}|w| d x \\
& \leq \varepsilon I+C_{\varepsilon} I_{5}+C \int_{U} K_{*} \cdot\left(\left|w^{2} Q\right|^{4 s}+\left|w^{2} Q\right|^{4 s+2}\right) \zeta^{2} d x+C I_{6} .
\end{aligned}
$$

Similarly,

$$
\begin{aligned}
J_{2} & \leq C \int_{U} \frac{K_{*}}{1+\left|\nabla w+w^{2} Q\right|}\left(\left|\nabla w+w^{2} Q\right|^{2 s+2}+\left|w^{2} Q\right|^{2 s+2}\right) \zeta^{2} w^{2}|Q| d x \\
& \leq C \int_{U} K_{*} \cdot\left(\left|\nabla w+w^{2} Q\right|^{2 s+1}+\left|w^{2} Q\right|^{2 s+2}\right) \zeta^{2} w^{2}|Q| d x \\
& \leq C \int_{U} K_{*} \cdot\left(|\nabla w|^{2 s+1}+\left|w^{2} Q\right|^{2 s+1}+\left|w^{2} Q\right|^{2 s+2}\right) \zeta^{2} w^{2}|Q| d x
\end{aligned}
$$

For $J_{3}$, neglecting the denominator in the integrand gives

$$
J_{3} \leq C \int_{U} K_{*}|\nabla w|^{2 s+1} \zeta^{2}|w|^{3}\left|Q^{\prime}\right| d x .
$$


Combining the above estimates for $J_{1}, J_{2}$ and $J_{3}$ yields

$$
I_{1} \leq \varepsilon I+C_{\varepsilon} I_{5}+C I_{6}+J_{4}+J_{5},
$$

where

$$
\begin{aligned}
& J_{4}=C \int_{U} K_{*}|\nabla w|^{2 s+1}\left(|Q|+|w|\left|Q^{\prime}\right|\right) \zeta^{2} w^{2} d x, \\
& J_{5}=C \int_{U} K_{*} \cdot\left(\left|w^{2} Q\right|^{4 s}+\left|w^{2} Q\right|^{4 s+2}\right) \zeta^{2} d x+C \int_{U} K_{*} \cdot\left(\left|w^{2} Q\right|^{2 s+1}+\left|w^{2} Q\right|^{2 s+2}\right) \zeta^{2} w^{2}|Q| d x .
\end{aligned}
$$

We estimate, by using Cauchy's inequality,

$$
J_{4} \leq \varepsilon I+C_{\varepsilon} \int_{U} K_{*}|\nabla w|^{2 s}\left(|Q|+|w|\left|Q^{\prime}\right|\right)^{2} \zeta^{2} w^{4} d x
$$

and, with $K_{*} \leq 1$

$$
\begin{aligned}
J_{5} & \leq C \int_{U}\left(\left|w^{2} Q\right|^{4 s}+\left|w^{2} Q\right|^{4 s+2}\right) \zeta^{2} d x+C \int_{U}\left(\left|w^{2} Q\right|^{2 s+1}+\left|w^{2} Q\right|^{2 s+2}\right) \zeta^{2} w^{2}|Q| d x \\
& =C \int_{U}\left(\left|w^{2} Q\right|^{4 s}+\left|w^{2} Q\right|^{4 s+2}+\left|w^{2} Q\right|^{2 s+2}+\left|w^{2} Q\right|^{2 s+3}\right) \zeta^{2} d x .
\end{aligned}
$$

Applying inequality (2.12) gives

$$
J_{5} \leq C \int_{U}\left(\left|w^{2} Q\right|^{2 s+2}+\left|w^{2} Q\right|^{4 s+2}\right) \zeta^{2} d x=C \int_{U}\left(1+\left|w^{2} Q\right|^{2 s}\right)\left|w^{2} Q\right|^{2 s+2} \zeta^{2} d x .
$$

Therefore,

$$
\begin{aligned}
I_{1} \leq & 2 \varepsilon I+C_{\varepsilon} I_{5}+C I_{6}+C_{\varepsilon} \int_{U} K_{*}|\nabla w|^{2 s}\left(|Q|+|w|\left|Q^{\prime}\right|\right)^{2} \zeta^{2} w^{4} d x \\
& +C \int_{U}\left(1+\left|w^{2} Q\right|^{2 s}\right)\left|w^{2} Q\right|^{2 s+2} \zeta^{2} d x .
\end{aligned}
$$

The terms $I_{2}, I_{3}, I_{4}$ can be bounded simply by

$$
\begin{aligned}
I_{2}+I_{3}+I_{4} \leq & \int_{U} K_{*}|\nabla w|^{2 s}|\Delta w| \zeta^{2}|w| d x+2 s \int_{U} K_{*}|\nabla w|^{2 s}\left|D^{2} w\right| \zeta^{2}|w| d x \\
& +2 \int_{U} K_{*}|\nabla w|^{2 s+1} \zeta|\nabla \zeta||w| d x \\
\leq & C \int_{U} K_{*}|\nabla w|^{2 s}\left|D^{2} w\right| \zeta^{2}|w| d x+2 \int_{U} K_{*}|\nabla w|^{2 s+1} \zeta|\nabla \zeta||w| d x
\end{aligned}
$$

Applying Cauchy's inequality to each integral gives

$$
I_{2}+I_{3}+I_{4} \leq\left(\varepsilon I+C_{\varepsilon} I_{5}\right)+\left(\varepsilon I+C_{\varepsilon} \int_{U} K_{*}|\nabla w|^{2 s}|\nabla \zeta|^{2} w^{2} d x\right) .
$$


Combining the estimates of $I_{1}$ and $I_{2}+I_{3}+I_{4}$, we have

$$
I \leq 4 \varepsilon I+C_{\varepsilon} I_{5}+C I_{6}+C_{\varepsilon} I_{7}+C \int_{U}\left(1+\left|w^{2} Q\right|^{2 s}\right)\left|w^{2} Q\right|^{2 s+2} \zeta^{2} d x
$$

where

$$
I_{7}=\int_{U} K_{*}|\nabla w|^{2 s}\left(|\nabla \zeta|^{2}+\left(|w|\left|Q^{\prime}\right|+|Q|\right)^{2} \zeta^{2} w^{2}\right) w^{2} d x
$$

Selecting $\varepsilon=1 / 8$, we obtain

$$
I \leq C\left(I_{5}+I_{6}+I_{7}\right)+C \int_{U}\left(1+\left|w^{2} Q\right|^{2 s}\right)\left|w^{2} Q\right|^{2 s+2} \zeta^{2} d x .
$$

In each integral of $I_{5}, I_{6}$ and $I_{7}$, we bound

$$
\zeta^{2} w^{2} \leq \zeta^{2} \sup _{\operatorname{supp} \zeta}\left(w^{2}\right), \quad|\nabla \zeta|^{2} w^{2} \leq|\nabla \zeta|^{2} \sup _{\operatorname{supp} \zeta}\left(w^{2}\right)
$$

It then follows (4.10) that

$$
\begin{aligned}
\int_{U} K_{*}|\nabla w|^{2 s+2} \zeta^{2} d x \leq & C \sup _{\operatorname{supp} \zeta}\left(w^{2}\right)\left\{\int_{U} K_{*}\left|D^{2} w\right|^{2}\left(|\nabla w|^{2 s-2}+1\right) \zeta^{2} d x\right. \\
& \left.+\int_{U} K_{*}|\nabla w|^{2 s}\left(|\nabla \zeta|^{2}+\left(|w|\left|Q^{\prime}\right|+|Q|\right)^{2} \zeta^{2} w^{2}\right) d x\right\} \\
& +C \int_{U}\left(1+\left|w^{2} Q\right|^{2 s}\right)\left|w^{2} Q\right|^{2 s+2} \zeta^{2} d x
\end{aligned}
$$

We compare $K_{*}$ in (4.9) with $K[w, Q]$ in (4.1). Because

$$
2^{-1}\left(1+\left|\nabla w+w^{2} Q\right|\right)^{2} \leq 1+\left|\nabla w+w^{2} Q\right|^{2} \leq\left(1+\left|\nabla w+w^{2} Q\right|\right)^{2}
$$

then

$$
K[w, Q](x) \leq K_{*}(x) \leq 2^{a / 2} K[w, Q](x) \quad \forall x \in U
$$

Applying the first, respectively second, inequality of (4.12) to the left-hand side, respectively right-hand side, of (4.11), we obtain inequality (4.7).

Now, consider the case $w, Q$ and $Q^{\prime}$ are bounded. By simple estimates of the last two integrals of (4.7) using the numbers $M_{w}, M_{Q}, \mu_{Q}$, and by using the following estimate

$$
|\nabla w|^{2 s-2}+1 \leq 2|\nabla w|^{2 s-2}+\operatorname{sgn}(s-1)
$$

for the first integral on the right-hand side of (4.7), we obtain inequality (4.8). 


\section{$5 \quad$ Gradient estimates (I)}

This section is focused on a priori estimates for the gradient of a solution $u(x, t)$ of the IBVP (1.23).

Hereafter, we fix $T>0$. Let $u(x, t)$ be a $C_{x, t}^{2,1}(\bar{U} \times(0, T]) \times C(\bar{U} \times[0, T])$ solution of (1.23), not necessarily non-negative.

In the estimates of the Lebesgue norms below, we will use the energy method. For that, it is convenient to shift the solution to a function vanishing at the boundary.

Let $\Psi(x, t)$ be the extension of $\psi(x, t)$ from $\Gamma \times(0, T]$ to $\bar{U} \times[0, T]$. It is assumed to have necessary regularity needed for calculations below. All our following estimates, as far as the boundary data is concerned, will be expressed in terms of $\Psi$. This will not lose the generality since we can always translate them into $\psi$-dependence estimates, see e.g. [20,25].

Define $\bar{u}=u-\Psi$ and $\bar{u}_{0}=u_{0}-\Psi$. We derive from (1.23) the equations for $\bar{u}$ :

$$
\begin{cases}\phi \bar{u}_{t}=\nabla \cdot\left(X(\Phi(x, t))-\phi \Psi_{t}\right. & \text { on } U \times(0, T] \\ \bar{u}(x, 0)=\bar{u}_{0}(x) & \text { on } U \\ \bar{u}(x, t)=0 & \text { on } \Gamma \times(0, T]\end{cases}
$$

where $\Phi(x, t)$ is the same notation as (3.1), i.e.,

$$
\Phi(x, t)=\nabla u(x, t)+u^{2}(x, t) \mathcal{Z}(x, t)=\nabla u(x, t)+W(x, t)
$$

with $W(x, t)=u^{2}(x, t) \mathcal{Z}(x, t)$.

The following "weight" function will play important roles in our statements and proofs:

$$
\mathcal{K}(x, t)=K[u(\cdot, t), \mathcal{Z}(\cdot, t)](x)=(1+|\Phi(x, t)|)^{-a} .
$$

We estimate the $L_{x, t}^{2-a}$-norm for $\nabla u$ in terms of the initial and boundary data. Define

$$
\begin{gathered}
M_{\mathcal{Z}}=\sup _{\bar{U} \times[0, T]}|\mathcal{Z}(x, t)| \\
\mathcal{E}_{0}=\int_{0}^{T} \int_{U}\left(\chi_{1}^{2(2+a)}|\nabla \Psi|^{2}+\phi \chi_{1}^{2}\left(\left|\Psi_{t}\right|^{2}+\Psi^{2}\right)\right) d x d t .
\end{gathered}
$$

Notation. In the remaining of this paper, the constant $C$ is positive and generic with varying values in different places. It depends on number $N$, the coefficients $a_{i}$ 's and powers $\alpha_{i}$ 's of the function $g$ in (1.9), the number $c_{*}$ in (2.6), and the set $U$. In sections 6 and 7 , it further depends on number $s$, the subsets $U^{\prime}, V, U_{k}$ 's of $U$. However, it does not depend on the initial and boundary data of $u$, the functions $\Phi(x, t), \mathcal{Z}(x, t)$, and numbers $T, T_{0}, t_{0}$, $\phi, \mathcal{R}, \chi_{*}, M_{*}$, whenever these are introduced. In particular, it is independent of the cut-off function $\zeta$ in Lemmas 6.1, 6.2, 6.7, Proposition 6.3, and inequality (7.1). 
Proposition 5.1. One has

$$
\begin{gathered}
\int_{0}^{T} \int_{U} \mathcal{K}|\nabla u|^{2} d x d t \leq C\left\{\chi_{1}^{2} \phi\left(\left\|\bar{u}_{0}\right\|_{L^{2}}^{2}+\|u\|_{L^{2}(U \times(0, T))}^{2}\right)\right. \\
\left.+\left(1+\chi_{1}\right)^{2(2+a)} M_{\mathcal{Z}}^{2}\|u\|_{L^{4}(U \times(0, T))}^{4}+\mathcal{E}_{0}\right\} \\
\int_{0}^{T} \int_{U}|\nabla u|^{2-a} d x d t \leq C\left\{\chi_{1}^{2} \phi\left(\left\|\bar{u}_{0}\right\|_{L^{2}}^{2}+\|u\|_{L^{2}(U \times(0, T))}^{2}\right)\right. \\
\left.+\left(1+\chi_{1}\right)^{2(2+a)}\left(T+M_{\mathcal{Z}}^{2}\|u\|_{L^{4}(U \times(0, T))}^{4}\right)+\mathcal{E}_{0}\right\} .
\end{gathered}
$$

Proof. In this proof, we denote $J=\int_{U} \Psi_{t} \bar{u} d x$. Multiplying the PDE (15.1) by $\bar{u}$, integrating over domain $U$ and using integration by parts, we have

$$
\begin{aligned}
\frac{\phi}{2} \frac{d}{d t} \int_{U} \bar{u}^{2} d x & =-\int_{U} X(\Phi) \cdot \nabla \bar{u} d x-\phi J \\
& =-\int_{U} X(\Phi) \cdot \Phi d x+\int_{U} X(\Phi) \cdot(\nabla \Psi+W) d x-\phi J
\end{aligned}
$$

By (2.17) and (2.19), we have

$$
\frac{\phi}{2} \frac{d}{d t} \int_{U} \bar{u}^{2} d x \leq-c_{4} \chi_{1}^{-2} \int_{U} \mathcal{K}|\Phi|^{2} d x+c_{2} \chi_{1}^{a} \int_{U} \mathcal{K}|\Phi|(|\nabla \Psi|+|W|) d x-\phi J .
$$

For the second integral on the right-hand side, applying Cauchy's inequality gives

$$
\begin{aligned}
\frac{\phi}{2} \frac{d}{d t} \int_{U} \bar{u}^{2} d x \leq & -c_{4} \chi_{1}^{-2} \int_{U} \mathcal{K}|\Phi|^{2} d x \\
& +\int_{U}\left[\varepsilon \mathcal{K}|\Phi|^{2}+C \varepsilon^{-1} \chi_{1}^{2 a} \mathcal{K}(|\nabla \Psi|+|W|)^{2}\right] d x-\phi J
\end{aligned}
$$

Choosing $\varepsilon=c_{4} \chi_{1}^{-2} / 2$, we obtain

$$
\frac{\phi}{2} \frac{d}{d t} \int_{U} \bar{u}^{2} d x \leq-\frac{c_{4} \chi_{1}^{-2}}{2} \int_{U} \mathcal{K}|\Phi|^{2} d x+C \chi_{1}^{2(1+a)} \int_{U}\left(|\nabla \Psi|^{2}+|W|^{2}\right) d x-\phi J .
$$

Note that

$$
|W| \leq M_{\mathcal{Z}} u^{2}
$$

Writing $\bar{u}$ in $J$ as $u-\Psi$, and using Cauchy's inequality, we have

$$
\phi|J| \leq \int_{U} \phi\left|\Psi_{t}\right|(|u|+|\Psi|) d x \leq \phi \int_{U}\left(u^{2}+\left|\Psi_{t}\right|^{2}+|\Psi|^{2}\right) d x
$$

Utilizing estimates (5.7) and (5.8) in (5.6), we derive

$$
\begin{aligned}
\frac{\phi}{2} \frac{d}{d t} \int_{U} \bar{u}^{2} d x+\frac{c_{4} \chi_{1}^{-2}}{2} \int_{U} \mathcal{K}|\Phi|^{2} d x \leq & C \chi_{1}^{2(1+a)} M_{\mathcal{Z}}^{2} \int_{U} u^{4} d x \\
& +C \chi_{1}^{2(1+a)} \int_{U}|\nabla \Psi|^{2} d x+\phi \int_{U}\left(u^{2}+\left|\Psi_{t}\right|^{2}+\Psi^{2}\right) d x
\end{aligned}
$$


Then integrating from 0 to $T$ gives

$$
\begin{aligned}
\int_{0}^{T} \int_{U} \mathcal{K}|\Phi|^{2} d x d t \leq & C \chi_{1}^{2} \phi\left\|\bar{u}_{0}\right\|_{L^{2}}^{2}+C \chi_{1}^{2(2+a)} M_{\mathcal{Z}}^{2} \int_{0}^{T} \int_{U} u^{4} d x d t+C \phi \chi_{1}^{2} \int_{0}^{T} \int_{U} u^{2} d x d t \\
& +C \mathcal{E}_{0} .
\end{aligned}
$$

For the left-hand side of (․5.9), we use inequalities (2.14) and (5.7) to have

$$
\mathcal{K}|\Phi|^{2}=\mathcal{K}|\nabla u+W|^{2} \geq 2^{-1} \mathcal{K}|\nabla u|^{2}-\mathcal{K}|W|^{2} \geq 2^{-1} \mathcal{K}|\nabla u|^{2}-M_{\mathcal{Z}}^{2} u^{4} .
$$

Combining this with (5.9) yields

$$
\begin{aligned}
\int_{0}^{T} \int_{U} \mathcal{K}|\nabla u|^{2} d x \leq & 2 \int_{0}^{T} \int_{U} \mathcal{K}|\Phi|^{2} d x d t+2 M_{\mathcal{Z}}^{2} \int_{0}^{T} \int_{U} u^{4} d x d t \\
\leq & C \chi_{1}^{2} \phi\left\|\bar{u}_{0}\right\|_{L^{2}}^{2}+C\left(1+\chi_{1}\right)^{2(2+a)} M_{\mathcal{Z}}^{2} \int_{0}^{T} \int_{U} u^{4} d x d t \\
& +C \phi \chi_{1}^{2} \int_{0}^{T} \int_{U} u^{2} d x d t+C \mathcal{E}_{0}
\end{aligned}
$$

which proves (5.4).

Using (4.3) and (5.7), we estimate the integrand on the left-hand side of (5.4) by

$$
\mathcal{K}|\nabla u|^{2} \geq 3^{-1}|\nabla u|^{2-a}-\left(1+|W|^{2}\right) \geq 3^{-1}|\nabla u|^{2-a}-\left(1+\left(u^{2} M_{\mathcal{Z}}\right)^{2}\right) .
$$

It results in

$$
\begin{aligned}
\int_{0}^{T} \int_{U}|\nabla u|^{2-a} d x d t \leq & 3 \int_{0}^{T} \int_{U} \mathcal{K}|\nabla u|^{2} d x+3 \int_{0}^{T} \int_{U}\left(1+M_{\mathcal{Z}}^{2} u^{4}\right) d x d t \\
\leq & C \chi_{1}^{2} \phi\left(\left\|\bar{u}_{0}\right\|_{L^{2}}^{2}+\|u\|_{L^{2}(U \times(0, T))}^{2}\right) \\
& +C\left(1+\chi_{1}\right)^{2(2+a)} \int_{0}^{T} \int_{U}\left(1+M_{\mathcal{Z}}^{2} u^{4}\right) d x d t+C \mathcal{E}_{0}
\end{aligned}
$$

which proves (5.5).

To have more specific estimates, we examine the bounds for the constituents of the PDE in (5.1). Note, from (1.22) and (2.7), that

$$
|\mathcal{Z}(x, t)| \leq \mathcal{G}+\Omega^{2}\left|\mathbf{J}^{2} x\right| \leq \mathcal{G}+\Omega^{2} r_{0}=\mathcal{G}\left(1+\Omega_{*}^{2}\right),
$$

where

$$
r_{0}=\max \{|x|: x \in \bar{U}\} \text { and } \Omega_{*}=\Omega \sqrt{r_{0} / \mathcal{G}}=\tilde{\Omega} \sqrt{r_{0} / \tilde{\mathcal{G}}} .
$$

Thus, the number $M_{\mathcal{Z}}$ in (5.2) can be bounded by

$$
M_{\mathcal{Z}} \leq \mathcal{G}\left(1+\Omega_{*}\right)^{2}
$$

Next, it is obvious that $D \mathcal{Z}(x, t)=\Omega^{2} \mathbf{J}^{2}$, hence, by (2.9),

$$
|D \mathcal{Z}(x, t)|=\mu_{\mathcal{Z}} \stackrel{\text { def }}{=} \sqrt{2} \Omega^{2}=\sqrt{2}\left(\mathcal{G} / r_{0}\right) \Omega_{*}^{2} .
$$


We rewrite $\mathcal{R}$ in (1.8) as

$$
\mathcal{R}=\frac{2 \rho_{*} \Omega}{\phi}=\frac{2 \rho_{*} \sqrt{\mathcal{G} / r_{0}}}{\phi} \Omega_{*}=\frac{2 \rho_{*} \sqrt{\tilde{\mathcal{G}} / r_{0}}}{\tilde{\phi}} \Omega_{*} .
$$

From (5.11), (5.12) and (5.13), we conveniently relate the upper bounds of $\mathcal{R}, M_{\mathcal{Z}}, \mu_{\mathcal{Z}}$ to a single parameter $\chi_{*}$ as follows

$$
\mathcal{R} \leq \chi_{*} \stackrel{\text { def }}{=} \max \left\{1, d_{*}\left(1+\Omega_{*}\right)\right\}, \quad M_{\mathcal{Z}} \leq \chi_{*}^{2}, \quad \mu_{\mathcal{Z}} \leq \chi_{*}^{2}
$$

where

$$
d_{*}=\sqrt{\mathcal{G} / r_{0}} \max \left\{\frac{2 \rho_{*}}{\phi}, \sqrt{r_{0}}, 2^{1 / 4}\right\} .
$$

The reason for (5.14), with the choice of $\chi_{*} \geq 1$, is to simplify many estimates that will be obtained later, and specify the dependence of those estimates on the parameters $d_{*}$ and $\Omega_{*}$.

Remark 5.2. A very common situation is that the rotation is about the vertical axis, then $\vec{k}= \pm(0,0,1)$, and

$$
\mathbf{J}= \pm\left(\begin{array}{ccc}
0 & -1 & 0 \\
1 & 0 & 0 \\
0 & 0 & 0
\end{array}\right), \quad \mathbf{J}^{2}=-\left(\begin{array}{lll}
1 & 0 & 0 \\
0 & 1 & 0 \\
0 & 0 & 0
\end{array}\right)
$$

Thanks to (5.15), we can, in this case, replace the $r_{0}$ in (5.10) with a smaller number, namely,

$$
r_{0}=\max \left\{\left(x_{1}^{2}+x_{2}^{2}\right)^{1 / 2}: x=\left(x_{1}, x_{2}, x_{3}\right) \in \bar{U}\right\}
$$

Definition 5.3. We will use the following quantities in our estimates throughout the paper:

$$
\begin{aligned}
& M_{*}=\sup _{U \times[0, T]}|u|, \quad \mathcal{E}_{*}=\int_{0}^{T} \int_{U}\left(|\nabla \Psi|^{2}+\phi\left|\Psi_{t}\right|^{2}+\phi \Psi^{2}\right) d x d t, \\
& \mathcal{N}_{0}=\phi\left\|\bar{u}_{0}\right\|_{L^{2}}^{2}+T M_{*}^{2}+\mathcal{E}_{*}, \quad \mathcal{N}_{*}=\phi\left\|\bar{u}_{0}\right\|_{L^{2}}^{2}+T+\mathcal{E}_{*}, \\
& \mathcal{N}_{2}=\phi\left(\left\|\bar{u}_{0}\right\|_{L^{2}}^{2}+\left\|\nabla u_{0}\right\|_{L^{2}}^{2}+T+\mathcal{E}_{*},\right. \\
& \mathcal{N}_{s}=\phi\left(\left\|\bar{u}_{0}\right\|_{L^{2}}^{2}+\left\|\nabla u_{0}\right\|_{L^{2}}^{2}+\left\|\nabla u_{0}\right\|_{L^{s}}^{s}\right)+T+\mathcal{E}_{*} \text { for } s>2 .
\end{aligned}
$$

The estimates obtained in the rest of this paper will depend on the quantities in Definition 5.3. Among those, only $M_{*}$ still depends on the solution $u$. However, this quantity can be bounded in terms of initial and boundary data by using different techniques. For instance, in our original problem, $u=\rho / \kappa \geq 0$, hence we have from Corollary 3.2 that $M_{*} \leq C M_{0}(T)$, see (3.8). Therefore, in the following, we say "the estimates are expressed in terms of the initial and boundary data" even when they contain $M_{*}$.

As stated in section 1, we will keep tracks of the dependence on certain physical parameters, particularly, the angular speed. Note, by (2.16) and (5.14), that

$$
\chi_{1} \leq C \chi_{*}
$$


With this and the fact $\chi_{*} \geq 1$, we compare $\mathcal{E}_{0}$ in $(5.3)$ with $\mathcal{E}_{*}$ by

$$
\mathcal{E}_{0} \leq C \chi_{*}^{2(2+a)} \mathcal{E}_{*} \text {. }
$$

It is clear that

$$
\begin{aligned}
& \mathcal{E}_{*} \leq \mathcal{N}_{0} \leq\left(M_{*}+1\right)^{2} \mathcal{N}_{*} \\
& \mathcal{E}_{*} \leq \mathcal{N}_{*} \leq \mathcal{N}_{2} \leq \mathcal{N}_{s} \text { for } s>2
\end{aligned}
$$

Theorem 5.4. One has

$$
\begin{gathered}
\int_{0}^{T} \int_{U} \mathcal{K}|\nabla u|^{2} d x d t \leq C\left\{\chi_{*}^{2} \phi\left\|\bar{u}_{0}\right\|_{L^{2}}^{2}+\chi_{*}^{2(4+a)} T M_{*}^{2}\left(M_{*}+1\right)^{2}+\chi_{*}^{2(2+a)} \mathcal{E}_{*}\right\}, \\
\int_{0}^{T} \int_{U}|\nabla u(x, t)|^{2-a} d x d t \leq C\left\{\chi_{*}^{2} \phi\left\|\bar{u}_{0}\right\|_{L^{2}}^{2}+\chi_{*}^{2(4+a)} T\left(M_{*}+1\right)^{4}+\chi_{*}^{2(2+a)} \mathcal{E}_{*}\right\} .
\end{gathered}
$$

Consequently, the following more concise estimates hold

$$
\begin{gathered}
\int_{0}^{T} \int_{U} \mathcal{K}|\nabla u|^{2} d x d t \leq C \chi_{*}^{2(4+a)}\left(M_{*}+1\right)^{2} \mathcal{N}_{0}, \\
\int_{0}^{T} \int_{U} \mathcal{K}|\nabla u|^{2} d x d t \leq C \chi_{*}^{2(4+a)}\left(M_{*}+1\right)^{4} \mathcal{N}_{*}, \\
\int_{0}^{T} \int_{U}|\nabla u(x, t)|^{2-a} d x d t \leq C \chi_{*}^{2(4+a)}\left(M_{*}+1\right)^{4} \mathcal{N}_{*} .
\end{gathered}
$$

Proof. By the definition of $M_{*}$, we obviously have

$$
|u| \leq M_{*} \text { on } \bar{U} \times[0, T]
$$

hence,

$$
\|u\|_{L^{2}(U \times(0, T))}^{2} \leq C T M_{*}^{2} \quad \text { and } \quad\|u\|_{L^{4}(U \times(0, T))}^{4} \leq C T M_{*}^{4} .
$$

Using (5.26), the relations (5.16), (5.17), and estimate of $M_{\mathcal{Z}}$ in (5.14) for the right-hand side of (5.4), we have

$$
\begin{aligned}
\int_{0}^{T} \int_{U} \mathcal{K}|\nabla u|^{2} d x d t & \leq C \chi_{*}^{2} \phi\left(\left\|\bar{u}_{0}\right\|_{L^{2}}^{2}+T M_{*}^{2}\right)+C\left(1+\chi_{*}\right)^{2(2+a)} \cdot \chi_{*}^{4} \cdot T M_{*}^{4}+C \chi_{*}^{2(2+a)} \mathcal{E}_{*} \\
& \leq C \chi_{*}^{2} \phi\left\|\bar{u}_{0}\right\|_{L^{2}}^{2}+C \chi_{*}^{2(2+a)+4} T M_{*}^{2}\left(1+M_{*}^{2}\right)+C \chi_{*}^{2(2+a)} \mathcal{E}_{*} .
\end{aligned}
$$

Then (5.20) and (5.22) follow. From (5.22), we infer (5.23) thanks to the last relation in (5.18).

Similarly, we have from (5.5) that

$$
\int_{0}^{T} \int_{U}|\nabla u(x, t)|^{2-a} d x d t \leq C\left\{\chi_{*}^{2} \phi\left(\left\|\bar{u}_{0}\right\|_{L^{2}}^{2}+T M_{*}^{2}\right)+\chi_{*}^{2(2+a)}\left(T+\chi_{*}^{4} \cdot T M_{*}^{4}\right)+\chi_{*}^{2(2+a)} \mathcal{E}_{*}\right\} .
$$

Then (5.21) and (5.24) follow.

We remark that while (5.21) gives a direct estimate for the $L^{2-a}$-norm, the alternative estimate in form of (5.20) prepares for the iterations in section 6 below.

Remark 5.5. From the point of view of pure PDE estimates, the right-hand side of (5.22) can be small, for a fixed $T>0$, while the right-hand side of (5.23) and (5.24) cannot. It is because $|u|,\left|\bar{u}_{0}\right|$, and $\mathcal{E}_{*}$ being small will make $\mathcal{N}_{0}$, but not $\mathcal{N}_{*}$, small. 


\section{Gradient estimates (II)}

In this section, we establish the interior $L^{s}$-estimate of $\nabla u$ for all $s>0$.

For the remainder of this paper, $\zeta$ always denotes a function in $C_{c}^{2}(U \times[0, T])$ that satisfies $0 \leq \zeta(x, t) \leq 1$ for all $(x, t)$. When such a function is specified, the quantity $\mathcal{D}_{s}$ is defined, for $s \geq 0$, by

$$
\mathcal{D}_{s}=\int_{U}\left|\nabla u_{0}(x)\right|^{2 s+2} \zeta^{2}(x, 0) d x .
$$

The next two lemmas 6.1 and 6.2 are the main technical steps for the later iterative estimates of the gradient.

Lemma 6.1. For any $s \geq 0$, one has

$$
\begin{aligned}
& \phi \sup _{t \in[0, T]} \int_{U}|\nabla u(x, t)|^{2 s+2} \zeta^{2}(x, t) d x, \\
& (s+1) c_{8} \chi_{1}^{-2} \int_{0}^{T} \int_{U} \mathcal{K}\left|D^{2} u\right|^{2}|\nabla u|^{2 s} \zeta^{2} d x d t \leq \phi \mathcal{D}_{s}+C I_{0},
\end{aligned}
$$

where

$$
\begin{aligned}
I_{0}= & \mu_{\mathcal{Z}}^{2}\left(1+\chi_{1}\right)^{2(1+a)} \int_{0}^{T} \int_{U} \mathcal{K}|\nabla u|^{2 s} u^{4} \zeta^{2} d x d t \\
& +\left(1+\chi_{1}\right)^{2(1+a)} \int_{0}^{T} \int_{U} \mathcal{K}|\nabla u|^{2 s+2}\left(M_{\mathcal{Z}}^{2} u^{2} \zeta^{2}+|\nabla \zeta|^{2}\right) d x d t+\int_{0}^{T} \int_{U}|\nabla u|^{2 s+2} \zeta\left|\zeta_{t}\right| d x d t .
\end{aligned}
$$

Consequently,

$$
\begin{aligned}
& \phi \sup _{t \in[0, T]} \int_{U}|\nabla u(x, t)|^{2 s+2} \zeta^{2}(x, t) d x \leq \phi \mathcal{D}_{s}+C J_{0}, \\
& \int_{0}^{T} \int_{U} \mathcal{K}\left|D^{2} u\right|^{2}|\nabla u|^{2 s} \zeta^{2} d x d t \leq C \chi_{*}^{2}\left(\phi \mathcal{D}_{s}+J_{0}\right),
\end{aligned}
$$

where

$$
\begin{aligned}
J_{0}= & \chi_{*}^{2(3+a)} \widetilde{M}^{4} \int_{0}^{T} \int_{U} \mathcal{K}|\nabla u|^{2 s} \zeta^{2} d x d t \\
& +\chi_{*}^{2(3+a)}(\widetilde{M}+1)^{2} \int_{0}^{T} \int_{U} \mathcal{K}|\nabla u|^{2 s+2}\left(\zeta^{2}+|\nabla \zeta|^{2}\right) d x d t+\int_{0}^{T} \int_{U}|\nabla u|^{2 s+2} \zeta\left|\zeta_{t}\right| d x d t,
\end{aligned}
$$

with

$$
\widetilde{M}=\sup \{|u(x, t)|:(x, t) \in \operatorname{supp} \zeta\} .
$$

Proof. For each $j=1,2,3$ and $t \in(0, T)$, let $\left\{\varphi_{n}(x)\right\}_{n=1}^{\infty}$ be a sequence in $C_{c}^{\infty}(U)$ that approximates $|\nabla u(x, t)|^{2 s} \partial_{j} u(x, t) \zeta^{2}(x, t)$ in $W_{0}^{1,2}(U)$. Multiplying equation (1.21) by $-\partial_{j} \varphi_{n}(x)$, 
integrating the resulting equation over $U$, and using the integration by parts twice for the right-hand side give

$$
\begin{aligned}
-\phi \int_{U} \frac{\partial u}{\partial t} \partial_{j} \varphi_{n} d x & =-\int_{U} \frac{\partial}{\partial x_{i}}\left[X_{i}(\Phi(x, t))\right] \partial_{j} \varphi_{n} d x=\int_{U} X_{i}(\Phi(x, t)) \partial_{i} \partial_{j} \varphi_{n} d x \\
& =-\int_{U} \frac{\partial}{\partial x_{j}}\left[X_{i}(\Phi(x, t))\right] \partial_{i} \varphi_{n} d x .
\end{aligned}
$$

Passing $n \rightarrow \infty$ and summing in $j$ yield

$$
-\phi \int_{U} \frac{\partial u}{\partial t} \nabla \cdot\left(|\nabla u|^{2 s} \nabla u \zeta^{2}\right) d x=-\int_{U} \frac{\partial}{\partial x_{j}}\left[X_{i}(\Phi(x, t))\right] \partial_{i}\left(|\nabla u|^{2 s} \partial_{j} u \zeta^{2}\right) d x .
$$

Performing integration by parts again for the left-hand side, we obtain

$$
\begin{aligned}
& \frac{\phi}{2 s+2} \frac{d}{d t} \int_{U}|\nabla u|^{2 s+2} \zeta^{2} d x \\
& =-\int_{U} \frac{\partial}{\partial x_{j}}\left[X_{i}(\Phi(x, t))\right] \partial_{i}\left(|\nabla u|^{2 s} \partial_{j} u \zeta^{2}\right) d x+\frac{1}{s+1} \int_{U}|\nabla u|^{2 s+2} \zeta \zeta_{t} d x \\
& =I_{1}+I_{2}+I_{3}+I_{4},
\end{aligned}
$$

where

$$
\begin{aligned}
I_{1} & =-\int_{U} \frac{\partial}{\partial x_{j}}\left[X_{i}(\Phi(x, t))\right] \partial_{j} \partial_{i} u|\nabla u|^{2 s} \zeta^{2} d x, \\
I_{2} & =-2 \int_{U} \frac{\partial}{\partial x_{j}}\left[X_{i}(\Phi(x, t))\right] \partial_{j} u|\nabla u|^{2 s} \zeta \partial_{i} \zeta d x, \\
I_{3} & =-2 s \int_{U} \frac{\partial}{\partial x_{j}}\left[X_{i}(\Phi(x, t))\right] \partial_{j} u\left(|\nabla u|^{2 s-2} \partial_{i} \partial_{l} u \partial_{l} u\right) \zeta^{2} d x, \\
I_{4} & =\frac{1}{s+1} \int_{U}|\nabla u|^{2 s+2} \zeta \zeta_{t} d x .
\end{aligned}
$$

For $i, j=1,2,3$, denote $X_{i j}^{\prime}(y)=\partial X_{i}(y) / \partial y_{j}$. By the second inequality of (2.36),

$$
\left|X_{i j}^{\prime}(\Phi(x, t))\right| \leq C\left(1+\chi_{1}\right)^{a} \mathcal{K}(x, t) .
$$

Let $I_{5}=\int_{U} \mathcal{K}\left|D^{2} u\right|^{2}|\nabla u|^{2 s} \zeta^{2} d x$.

Estimation of $I_{1}$. We have

$$
\begin{aligned}
I_{1} & =-\int_{U} X_{i m}^{\prime}(\Phi)\left(\partial_{m} \partial_{j} u+\partial_{j} \mathcal{Z}_{m} u^{2}+2 u \partial_{j} u \mathcal{Z}_{m}\right) \partial_{j} \partial_{i} u|\nabla u|^{2 s} \zeta^{2} d x \\
& =I_{1,1}+I_{1,2}+I_{1,3},
\end{aligned}
$$

where

$$
\begin{aligned}
& I_{1,1}=-\int_{U} D\left(\partial_{j} u\right) X^{\prime}(\Phi) \nabla\left(\partial_{j} u\right)|\nabla u|^{2 s} \zeta^{2} d x, \\
& I_{1,2}=-\int_{U} X_{i m}^{\prime}(\Phi) \partial_{j} \mathcal{Z}_{m} u^{2} \partial_{j} \partial_{i} u|\nabla u|^{2 s} \zeta^{2} d x, \\
& I_{1,3}=-2 \int_{U} X_{i m}^{\prime}(\Phi) u \partial_{j} u \mathcal{Z}_{m} \partial_{j} \partial_{i} u|\nabla u|^{2 s} \zeta^{2} d x .
\end{aligned}
$$


To estimate $I_{1,1}$, by applying (2.37) to $y=\Phi, \xi=\nabla\left(\partial_{j} u\right)$, we have

$$
I_{1,1} \leq-c_{8} \chi_{1}^{-2} \int_{U} \mathcal{K}\left(\sum_{j=1}^{n}\left|\nabla\left(\partial_{j} u\right)\right|^{2}\right)|\nabla u|^{2 s} \zeta^{2} d x=-c_{8} \chi_{1}^{-2} I_{5}
$$

To estimate $I_{1,2}$, using inequality (6.7) to estimate $\left|X_{i m}^{\prime}(\Phi)\right|$, identity (15.12) for $\left|\partial_{j} \mathcal{Z}_{m}\right|$, and then applying the Cauchy inequality to $u^{2}\left|D^{2} u\right|$, we obtain

$$
\begin{aligned}
\left|I_{1,2}\right| & \leq C \int_{U}\left(1+\chi_{1}\right)^{a} \mathcal{K} \mu_{\mathcal{Z}} u^{2}\left|D^{2} u\right||\nabla u|^{2 s} \zeta^{2} d x \\
& \leq \varepsilon I_{5}+C \varepsilon^{-1}\left(1+\chi_{1}\right)^{2 a} \mu_{\mathcal{Z}}^{2} \int_{U} \mathcal{K} u^{4}|\nabla u|^{2 s} \zeta^{2} d x
\end{aligned}
$$

Similarly, we estimate $I_{1,3}$ by

$$
\begin{aligned}
\left|I_{1,3}\right| & \leq C\left(1+\chi_{1}\right)^{a} \int_{U} \mathcal{K} M_{\mathcal{Z}} u\left|D^{2} u\right||\nabla u|^{2 s+1} \zeta^{2} d x \\
& \leq \varepsilon I_{5}+C \varepsilon^{-1}\left(1+\chi_{1}\right)^{2 a} M_{\mathcal{Z}}^{2} \int_{U} \mathcal{K} u^{2}|\nabla u|^{2 s+2} \zeta^{2} d x .
\end{aligned}
$$

Summing up, we obtain

$$
\begin{aligned}
I_{1} \leq & \left(2 \varepsilon-c_{8} \chi_{1}^{-2}\right) I_{5}+C \varepsilon^{-1}\left(1+\chi_{1}\right)^{2 a} \mu_{\mathcal{Z}}^{2} \int_{U} \mathcal{K} u^{4}|\nabla u|^{2 s} \zeta^{2} d x \\
& +C \varepsilon^{-1}\left(1+\chi_{1}\right)^{2 a} M_{\mathcal{Z}}^{2} \int_{U} \mathcal{K} u^{2}|\nabla u|^{2 s+2} \zeta^{2} d x .
\end{aligned}
$$

Estimation of $I_{2}$. We calculate

$$
\begin{aligned}
I_{2} & =-2 \int_{U} X_{i m}^{\prime}(\Phi)\left(\partial_{m} \partial_{j} u+\partial_{j} \mathcal{Z}_{m} u^{2}+2 u \partial_{j} u \mathcal{Z}_{m}\right) \partial_{j} u|\nabla u|^{2 s} \zeta \partial_{i} \zeta d x \\
& =I_{2,1}+I_{2,2}+I_{2,3}
\end{aligned}
$$

where the integral is split along the sum $\partial_{m} \partial_{j} u+\partial_{j} \mathcal{Z}_{m} u^{2}+2 u \partial_{j} u \mathcal{Z}_{m}$.

Using (6.7) and Cauchy's inequality gives

$$
\begin{aligned}
\left|I_{2,1}\right| & \leq C\left(1+\chi_{1}\right)^{a} \int_{U} \mathcal{K}\left|D^{2} u\right||\nabla u|^{2 s+1} \zeta|\nabla \zeta| d x \\
& \leq \varepsilon I_{5}+C \varepsilon^{-1}\left(1+\chi_{1}\right)^{2 a} \int_{U} \mathcal{K}|\nabla u|^{2 s+2}|\nabla \zeta|^{2} d x
\end{aligned}
$$

Using (6.7), (5.12) and (5.2), we obtain

$$
\begin{aligned}
\left|I_{2,2}\right| & \leq C\left(1+\chi_{1}\right)^{a} \int_{U} \mathcal{K}|D \mathcal{Z}| u^{2}|\nabla u|^{2 s+1} \zeta|\nabla \zeta| d x \\
& \leq C\left(1+\chi_{1}\right)^{a} \mu_{\mathcal{Z}} \int_{U} \mathcal{K} u^{2}|\nabla u|^{2 s+1} \zeta|\nabla \zeta| d x
\end{aligned}
$$


and

$$
\begin{aligned}
\left|I_{2,3}\right| & \leq C\left(1+\chi_{1}\right)^{a} \int_{U} \mathcal{K}|\mathcal{Z}| u|\nabla u|^{2 s+2} \zeta|\nabla \zeta| d x \\
& \leq C\left(1+\chi_{1}\right)^{a} M_{\mathcal{Z}} \int_{U} \mathcal{K} u|\nabla u|^{2 s+2} \zeta|\nabla \zeta| d x
\end{aligned}
$$

Thus, we have

$$
\begin{aligned}
I_{2} & \leq \varepsilon I_{5}+C \varepsilon^{-1}\left(1+\chi_{1}\right)^{2 a} \int_{U} \mathcal{K}|\nabla u|^{2 s+2}|\nabla \zeta|^{2} d x \\
& +C\left(1+\chi_{1}\right)^{a} \mu_{\mathcal{Z}} \int_{U} \mathcal{K} u^{2}|\nabla u|^{2 s+1} \zeta|\nabla \zeta| d x+C\left(1+\chi_{1}\right)^{a} M_{\mathcal{Z}} \int_{U} \mathcal{K} u|\nabla u|^{2 s+2} \zeta|\nabla \zeta| d x
\end{aligned}
$$

Estimation of $I_{3}$. Using similar calculations to those for $I_{2}$, we have

$$
\begin{aligned}
I_{3} & =-2 s \int_{U} X_{m i}^{\prime}(\Phi)\left(\partial_{m} \partial_{j} u+\partial_{j} \mathcal{Z}_{m} u^{2}+2 u \partial_{j} u \mathcal{Z}_{m}\right) \cdot \partial_{j} u\left(|\nabla u|^{2 s-2} \partial_{i} \partial_{l} u \partial_{l} u\right) \zeta^{2} d x \\
& =I_{3,1}+I_{3,2}+I_{3,3} .
\end{aligned}
$$

where the integral, again, is split along the sum $\partial_{m} \partial_{j} u+\partial_{j} \mathcal{Z}_{m} u^{2}+2 u \partial_{j} u \mathcal{Z}_{m}$.

Rewriting $I_{3,1}$ and applying (2.37) to $y=\Phi$ and $\xi=\frac{1}{2} \nabla\left(|\nabla u|^{2}\right)$, we have

$$
\begin{aligned}
I_{3,1} & =-2 s \int_{U} X_{i m}^{\prime}(\Phi) \cdot\left[\partial_{m} \partial_{j} u \cdot \partial_{j} u\right] \cdot\left[\partial_{i} \partial_{l} \cdot u \partial_{l} u\right] \cdot|\nabla u|^{2 s-2} \zeta^{2} d x \\
& \left.=-2 s \int_{U} X_{i m}^{\prime}(\Phi)\left[\frac{1}{2} \partial_{m}|\nabla u|^{2}\right]\left[\frac{1}{2} \partial_{i}|\nabla u|^{2}\right)\right] \cdot|\nabla u|^{2 s-2} \zeta^{2} d x \\
& \leq-2 s c_{8} \chi_{1}^{-2} \int_{U} \mathcal{K}\left|\frac{1}{2} \nabla\left(|\nabla u|^{2}\right)\right|^{2}|\nabla u|^{2 s-2} \zeta^{2} d x \leq 0 .
\end{aligned}
$$

We estimate $I_{3,2}$ and $I_{3,3}$ similarly to $I_{2,1}, I_{2,2}, I_{2,3}$, and obtain

$$
\begin{aligned}
\left|I_{3,2}\right| & \leq C\left(1+\chi_{1}\right)^{a} \int_{U} \mathcal{K}|D \mathcal{Z}| u^{2}|\nabla u|^{2 s}\left|D^{2} u\right| \zeta^{2} d x \\
& \leq \varepsilon I_{5}+C \varepsilon^{-1}\left(1+\chi_{1}\right)^{2 a} \mu_{\mathcal{Z}}^{2} \int_{U} \mathcal{K} u^{4}|\nabla u|^{2 s} \zeta^{2} d x
\end{aligned}
$$

and

$$
\begin{aligned}
\left|I_{3,3}\right| & \leq C\left(1+\chi_{1}\right)^{a} M_{\mathcal{Z}} \int_{U} \mathcal{K} u|\nabla u|^{2 s+1}\left|D^{2} u\right| \zeta^{2} d x \\
& \leq \varepsilon I_{5}+C \varepsilon^{-1}\left(1+\chi_{1}\right)^{2 a} M_{\mathcal{Z}}^{2} \int_{U} \mathcal{K} u^{2}|\nabla u|^{2 s+2} \zeta^{2} d x
\end{aligned}
$$

Therefore,

$$
\begin{aligned}
I_{3} \leq & 2 \varepsilon I_{5}+C \varepsilon^{-1}\left(1+\chi_{1}\right)^{2 a} \mu_{\mathcal{Z}}^{2} \int_{U} \mathcal{K} u^{4}|\nabla u|^{2 s} \zeta^{2} d x \\
& +C \varepsilon^{-1}\left(1+\chi_{1}\right)^{2 a} M_{\mathcal{Z}}^{2} \int_{U} \mathcal{K} u^{2}|\nabla u|^{2 s+2} \zeta^{2} d x .
\end{aligned}
$$


Combining (6.6) with the estimates (6.8), (6.9) and (6.10), we have

$$
\begin{aligned}
& \frac{\phi}{2 s+2} \frac{d}{d t} \int_{U}|\nabla u|^{2 s+2} \zeta^{2} d x+\left(c_{8} \chi_{1}^{-2}-5 \varepsilon\right) I_{5} \leq C \varepsilon^{-1}\left(1+\chi_{1}\right)^{2 a} \mu_{\mathcal{Z}}^{2} \int_{U} \mathcal{K} u^{4}|\nabla u|^{2 s} \zeta^{2} d x \\
& +C \varepsilon^{-1}\left(1+\chi_{1}\right)^{2 a} M_{\mathcal{Z}}^{2} \int_{U} \mathcal{K} u^{2}|\nabla u|^{2 s+2} \zeta^{2} d x+C \varepsilon^{-1}\left(1+\chi_{1}\right)^{2 a} \int_{U} \mathcal{K}|\nabla u|^{2 s+2}|\nabla \zeta|^{2} d x \\
& +C\left(1+\chi_{1}\right)^{a} \mu_{\mathcal{Z}} \int_{U} \mathcal{K}|u|^{2}|\nabla u|^{2 s+1} \zeta|\nabla \zeta| d x+C\left(1+\chi_{1}\right)^{a} M_{\mathcal{Z}} \int_{U} \mathcal{K}|u||\nabla u|^{2 s+2} \zeta|\nabla \zeta| d x+I_{4} .
\end{aligned}
$$

Using the Cauchy inequality, we have, for the fourth integral on the right-hand side,

$$
\left(1+\chi_{1}\right)^{a} \mu_{\mathcal{Z}}|u|^{2}|\nabla u|^{2 s+1} \zeta|\nabla \zeta| \leq \frac{1}{2}\left[\varepsilon^{-1}\left(1+\chi_{1}\right)^{2 a} \mu_{\mathcal{Z}}^{2} u^{4}|\nabla u|^{2 s} \zeta^{2}+\varepsilon|\nabla u|^{2 s+2}|\nabla \zeta|^{2}\right],
$$

and, for the fifth integral on the right-hand side,

$$
\left(1+\chi_{1}\right)^{a} M_{\mathcal{Z}}|u| \zeta|\nabla \zeta| \leq \frac{1}{2}\left[\varepsilon^{-1}\left(1+\chi_{1}\right)^{2 a} M_{\mathcal{Z}}^{2} u^{2} \zeta^{2}+\varepsilon|\nabla \zeta|^{2}\right] .
$$

Therefore, we obtain

$$
\begin{aligned}
& \frac{\phi}{2 s+2} \frac{d}{d t} \int_{U}|\nabla u|^{2 s+2} \zeta^{2} d x+\left(c_{8} \chi_{1}^{-2}-5 \varepsilon\right) I_{5} \\
& \leq C \varepsilon^{-1}\left(1+\chi_{1}\right)^{2 a} \mu_{\mathcal{Z}}^{2} \int_{U} \mathcal{K}|\nabla u|^{2 s} u^{4} \zeta^{2} d x+C \varepsilon^{-1}\left(1+\chi_{1}\right)^{2 a} M_{\mathcal{Z}}^{2} \int_{U} \mathcal{K} u^{2}|\nabla u|^{2 s+2} \zeta^{2} d x \\
& \quad+C\left(\varepsilon^{-1}\left(1+\chi_{1}\right)^{2 a}+\varepsilon\right) \int_{U} \mathcal{K}|\nabla u|^{2 s+2}|\nabla \zeta|^{2} d x+C \int_{U}|\nabla u|^{2 s+2} \zeta\left|\zeta_{t}\right| d x .
\end{aligned}
$$

Choosing $\varepsilon=c_{8} \chi_{1}^{-2} / 10$, and integrating (6.11) in time, we get

$$
\phi \sup _{t \in[0, T]} \int_{U}|\nabla u(x, t)|^{2 s+2} \zeta^{2}(x, t) d x \text { and }(s+1) c_{8} \chi_{1}^{-2} \int_{0}^{T} \int_{U} \mathcal{K}\left|D^{2} u\right|^{2}|\nabla u|^{2 s} \zeta^{2} d x d t
$$

are bounded from above by

$$
\begin{aligned}
& \phi \int_{U}\left|\nabla u_{0}(x)\right|^{2 s+2} \zeta^{2}(x, 0) d x+C \mu_{\mathcal{Z}}^{2}\left(1+\chi_{1}\right)^{2(1+a)} \int_{0}^{T} \int_{U} \mathcal{K}|\nabla u|^{2 s} u^{4} \zeta^{2} d x d t \\
& \quad+C\left(\left(1+\chi_{1}\right)^{2(1+a)}+\varepsilon\right) \int_{0}^{T} \int_{U} \mathcal{K}|\nabla u|^{2 s+2}\left(M_{\mathcal{Z}}^{2} u^{2} \zeta^{2}+|\nabla \zeta|^{2}\right) d x d t \\
& \quad+C \int_{0}^{T} \int_{U}|\nabla u|^{2 s+2} \zeta\left|\zeta_{t}\right| d x d t .
\end{aligned}
$$

Then using the fact $\varepsilon \leq c_{8} \chi_{0}^{-2} / 10 \leq C$ for the last $\varepsilon$, we obtain (6.2).

We now estimate $I_{0}$ further. We use (5.14), (5.16) to estimate $M_{\mathcal{Z}}, \mu_{\mathcal{Z}}, \chi_{1}$, and note, by (6.5), that $u^{m} \zeta^{2} \leq \widetilde{M}^{m} \zeta^{2}$ for $m=2$, 4. With these estimates, we have

$$
I_{0} \leq C J_{0}
$$

Hence, (6.3) directly follows the first estimate (6.2).

Similarly, multiplying the second estimate in (6.2) by $(s+1)^{-1} c_{8}^{-1} \chi_{1}^{2}$, then using (5.16) and (6.12), we obtain (6.4). 
Next, we combine Lemma 6.1 with the embedding in Theorem 4.2 to derive a bootstrapping estimate.

Lemma 6.2. If $s \geq 0$ then

$$
\int_{0}^{T} \int_{U} \mathcal{K}|\nabla u|^{2 s+4} \zeta^{2} d x d t+M_{*}^{2} \int_{0}^{T} \int_{U} \mathcal{K}\left|D^{2} u\right|^{2}|\nabla u|^{2 s} \zeta^{2} d x d t \leq C\left(I_{*}+J_{*}\right),
$$

where

$$
\begin{aligned}
I_{*}= & \chi_{*}^{2} M_{*}^{2} \phi \mathcal{D}_{s}+T \chi_{*}^{4(2 s+3)} M_{*}^{6}\left(M_{*}+1\right)^{8 s+6} \\
& +\chi_{*}^{2(4+a)} M_{*}^{2}\left(M_{*}+1\right)^{4} \int_{0}^{T} \int_{U} \mathcal{K}|\nabla u|^{2 s+2}\left(\zeta^{2}+|\nabla \zeta|^{2}\right) d x d t \\
& +\operatorname{sgn}(s) M_{*}^{2} \int_{0}^{T} \int_{U} \mathcal{K}\left|D^{2} u\right|^{2} \zeta^{2} d x d t
\end{aligned}
$$

and

$$
\begin{aligned}
J_{*}= & T \chi_{*}^{4(1+a)} M_{*}^{4}\left(M_{*}+1\right)^{4 a} \sup \left|\zeta_{t}\right|^{2} \\
& +\chi_{*}^{4(1+a \cdot \operatorname{sgn}(s))} M_{*}^{4}\left(M_{*}+1\right)^{4 a \cdot \operatorname{sgn}(s)} \int_{0}^{T} \int_{U} \mathcal{K}|\nabla u|^{2 s+2}\left|\zeta_{t}\right|^{2} d x d t .
\end{aligned}
$$

Proof. Denote

$$
\begin{array}{ll}
\alpha=\int_{0}^{T} \int_{U} \mathcal{K}|\nabla u|^{2 s+4} \zeta^{2} d x d t, & \gamma=\int_{0}^{T} \int_{U}|\nabla u|^{2 s+2} \zeta\left|\zeta_{t}\right| d x d t \\
\beta=\int_{0}^{T} \int_{U} \mathcal{K}\left|D^{2} u\right|^{2}|\nabla u|^{2 s} \zeta^{2} d x d t, & \beta_{0}=\int_{0}^{T} \int_{U} \mathcal{K}\left|D^{2} u\right|^{2} \zeta^{2} d x d t
\end{array}
$$

For $s \geq 0$, by applying (4.8) to $w(x)=u(x, t), Q(x)=\mathcal{Z}(x, t), s:=s+1$, and then integrating in $t$ from 0 to $T$, we have

$$
\begin{aligned}
\alpha \leq & C M_{*}^{2} \beta+C M_{*}^{2}\left(1+\left(M_{*} \mu_{\mathcal{Z}}+M_{\mathcal{Z}}\right)^{2} M_{*}^{2}\right) \int_{0}^{T} \int_{U} \mathcal{K}|\nabla u|^{2 s+2}\left(|\nabla \zeta|^{2}+\zeta^{2}\right) d x d t \\
& +C \operatorname{sgn}(s) M_{*}^{2} \beta_{0}+C T\left(M_{*}^{2} M_{\mathcal{Z}}\right)^{2 s+4}\left(1+M_{*}^{2} M_{\mathcal{Z}}\right)^{2 s+2} .
\end{aligned}
$$

Using (5.14) for upper bounds of $M_{\mathcal{Z}}$ and $\mu_{\mathcal{Z}}$, we have

$$
\begin{aligned}
\alpha \leq & C M_{*}^{2} \beta+C \chi_{*}^{4} M_{*}^{2}\left(M_{*}+1\right)^{4} \int_{0}^{T} \int_{U} \mathcal{K}|\nabla u|^{2 s+2}\left(|\nabla \zeta|^{2}+\zeta^{2}\right) d x d t \\
& +C \operatorname{sgn}(s) M_{*}^{2} \beta_{0}+C T \chi_{*}^{4(2 s+3)} M_{*}^{4(s+2)}\left(M_{*}+1\right)^{4(s+1)} .
\end{aligned}
$$

We estimate $\beta$ by using (6.4) and the fact $\widetilde{M} \leq M_{*}$ to have

$$
\begin{aligned}
C M_{*}^{2} \beta \leq & C \chi_{*}^{2} M_{*}^{2} \phi \mathcal{D}_{s}+C \chi_{*}^{2(4+a)} M_{*}^{6} \int_{0}^{T} \int_{U} \mathcal{K}|\nabla u|^{2 s} \zeta^{2} d x d t \\
& +C \chi_{*}^{2(4+a)} M_{*}^{2}\left(M_{*}+1\right)^{2} \int_{0}^{T} \int_{U} \mathcal{K}|\nabla u|^{2 s+2}\left(\zeta^{2}+|\nabla \zeta|^{2}\right) d x d t+C \chi_{*}^{2} M_{*}^{2} \gamma
\end{aligned}
$$


Thus,

$$
\begin{aligned}
2 \alpha+M_{*}^{2} \beta \leq & C \chi_{*}^{2} M_{*}^{2} \phi \mathcal{D}_{s}+C \chi_{*}^{2(4+a)} M_{*}^{6} \int_{0}^{T} \int_{U} \mathcal{K}|\nabla u|^{2 s} \zeta^{2} d x d t \\
& +C \chi_{*}^{2(4+a)} M_{*}^{2}\left(M_{*}+1\right)^{4} \int_{0}^{T} \int_{U} \mathcal{K}|\nabla u|^{2 s+2}\left(\zeta^{2}+|\nabla \zeta|^{2}\right) d x d t \\
& +C \operatorname{sgn}(s) M_{*}^{2} \beta_{0}+C T \chi_{*}^{4(2 s+3)} M_{*}^{4(s+2)}\left(M_{*}+1\right)^{4(s+1)}+C \chi_{*}^{2} M_{*}^{2} \gamma .
\end{aligned}
$$

For the second term on the right-hand side of (6.14), the integral is bounded by

$$
\int_{0}^{T} \int_{U} \mathcal{K}|\nabla u|^{2 s} \zeta^{2} d x d t \leq \int_{0}^{T} \int_{U} \mathcal{K}\left(|\nabla u|^{2 s+2}+1\right) \zeta^{2} d x d t \leq \int_{0}^{T} \int_{U} \mathcal{K}|\nabla u|^{2 s+2} \zeta^{2} d x d t+C T .
$$

Combining this with the third term on the right-hand side of (6.14) gives

$$
\begin{aligned}
2 \alpha & +M_{*}^{2} \beta \leq C \chi_{*}^{2} M_{*}^{2} \phi \mathcal{D} s+C \chi_{*}^{2(4+a)} M_{*}^{2}\left(M_{*}+1\right)^{4} \int_{0}^{T} \int_{U} \mathcal{K}|\nabla u|^{2 s+2}\left(\zeta^{2}+|\nabla \zeta|^{2}\right) d x d t \\
& +C \operatorname{sgn}(s) M_{*}^{2} \beta_{0}+C T \chi_{*}^{4(2 s+3)} M_{*}^{4(s+2)}\left(M_{*}+1\right)^{4(s+1)}+C T \chi_{*}^{2(4+a)} M_{*}^{6}+C \chi_{*}^{2} M_{*}^{2} \gamma .
\end{aligned}
$$

As far as the two $T$-terms in the last inequality are concerned, the first one has

$$
M_{*}^{4(s+2)}\left(M_{*}+1\right)^{4(s+1)} \leq M_{*}^{6}\left(M_{*}+1\right)^{8 s+6},
$$

while the second one has

$$
\chi_{*}^{2(4+a)} \leq \chi_{*}^{10} \leq \chi_{*}^{4(2 s+3)}
$$

Therefore,

$$
2 \alpha+M_{*}^{2} \beta \leq C I_{*}+C \chi_{*}^{2} M_{*}^{2} \gamma .
$$

We estimate the last term by using Cauchy's inequality to obtain

$$
\begin{aligned}
C \chi_{*}^{2} M_{*}^{2} \gamma & \leq \int_{0}^{T} \int_{U} \mathcal{K}|\nabla u|^{2 s+4} \zeta^{2} d x d t+C \chi_{*}^{4} M_{*}^{4} \int_{0}^{T} \int_{U} \mathcal{K}^{-1}|\nabla u|^{2 s}\left|\zeta_{t}\right|^{2} d x d t \\
& =\alpha+C \chi_{*}^{4} M_{*}^{4} \int_{0}^{T} \int_{U} \mathcal{K}|\nabla u|^{2 s} \mathcal{K}^{-2}\left|\zeta_{t}\right|^{2} d x d t
\end{aligned}
$$

In the last integral, we have

$$
\begin{aligned}
|\nabla u|^{2 s} \mathcal{K}^{-2} & \leq|\nabla u|^{2 s}\left(1+|\nabla u|+M_{*}^{2} \chi_{*}^{2}\right)^{2 a} \\
& \leq C\left(|\nabla u|^{2 s}+|\nabla u|^{2 s+2 a}+M_{*}^{4 a} \chi_{*}^{4 a}|\nabla u|^{2 s}\right) \\
& \leq C\left[1+|\nabla u|^{2 s+2}+M_{*}^{4 a} \chi_{*}^{4 a}\left(1+\operatorname{sgn}(s)|\nabla u|^{2 s+2}\right)\right],
\end{aligned}
$$

which can be conveniently rewritten as

$$
|\nabla u|^{2 s} \mathcal{K}^{-2} \leq C\left(M_{*}+1\right)^{4 a} \chi_{*}^{4 a}+C\left(M_{*}+1\right)^{4 a \cdot \operatorname{sgn}(s)} \chi_{*}^{4 a \cdot \operatorname{sgn}(s)}|\nabla u|^{2 s+2} .
$$


Thus,

$$
\begin{aligned}
C \chi_{*}^{2} M_{*}^{2} \gamma \leq & \alpha+C \chi_{*}^{4(1+a \cdot \operatorname{sgn}(s))} M_{*}^{4}\left(M_{*}+1\right)^{4 a \cdot \operatorname{sgn}(s)} \int_{0}^{T} \int_{U} \mathcal{K}|\nabla u|^{2 s+2}\left|\zeta_{t}\right|^{2} d x d t \\
& +C \chi_{*}^{4(1+a)} M_{*}^{4}\left(M_{*}+1\right)^{4 a} \int_{0}^{T} \int_{U}\left|\zeta_{t}\right|^{2} d x d t \leq \alpha+C J_{*} .
\end{aligned}
$$

Combining (6.15) and (6.16) yields

$$
2 \alpha+M_{*}^{2} \beta \leq C I_{*}+\alpha+C J_{*} \text {, which implies } \alpha+M_{*}^{2} \beta \leq C\left(I_{*}+J_{*}\right) .
$$

We have proved (6.13).

As one can see from (6.13) that the integral of higher power $2 s+4$ of $|\nabla u|$, with the weight $\mathcal{K}(x, t)$, can be bounded by the corresponding integral of lower power $2 s+2$. However,

it still involves a second order term, which is the last integral of $I_{*}$. This term, as it turns out, can be estimated in (6.17) below.

\subsection{Estimates for the $L_{x, t}^{4-a}$-norm}

We start using inequality (6.13) with the smallest possible value for $s$, i.e., $s=0$. It will result in the $\mathcal{K}$-weighted $L_{x, t}^{2}$-estimate, and, consequently, the $L_{x, t}^{4-a}$ estimate for $|\nabla u|$.

Proposition 6.3. One has

$$
\begin{aligned}
& \int_{0}^{T} \int_{U} \mathcal{K}|\nabla u|^{4} \zeta^{2} d x d t+M_{*}^{2} \int_{0}^{T} \int_{U} \mathcal{K}\left|D^{2} u\right|^{2} \zeta^{2} d x d t \\
& \leq C\left(1+\sup |\nabla \zeta|^{2}+\sup \left|\zeta_{t}\right|^{2}\right)\left\{\chi_{*}^{2(5+a)} M_{*}^{2}\left(M_{*}+1\right)^{4} \phi\left(\left\|\bar{u}_{0}\right\|_{L^{2}}^{2}+\mathcal{D}_{0}\right)\right. \\
& \left.\quad+\chi_{*}^{4(4+a)} T M_{*}^{4}\left(M_{*}+1\right)^{8}+\chi_{*}^{4(3+a)} M_{*}^{2}\left(M_{*}+1\right)^{4} \mathcal{E}_{*}\right\}
\end{aligned}
$$

Proof. Denote by $I$ the sum on the left-hand side of (6.17). It follows (6.13) with $s=0$ that

$$
\begin{aligned}
I \leq & C \chi_{*}^{2} M_{*}^{2} \phi \mathcal{D}_{0}+C T \cdot\left[\chi_{*}^{12} M_{*}^{6}\left(M_{*}+1\right)^{6}+\chi_{*}^{4(1+a)} M_{*}^{4}\left(M_{*}+1\right)^{4 a} \sup \left|\zeta_{t}\right|^{2}\right] \\
& +C \chi_{*}^{2(4+a)} M_{*}^{2}\left(M_{*}+1\right)^{4} \int_{0}^{T} \int_{U} \mathcal{K}|\nabla u|^{2}\left(\zeta^{2}+|\nabla \zeta|^{2}\right) d x d t \\
& +C \chi_{*}^{4} M_{*}^{4} \int_{0}^{T} \int_{U} \mathcal{K}|\nabla u|^{2}\left|\zeta_{t}\right|^{2} d x d t
\end{aligned}
$$

The second term on the right-hand side is bounded by

$$
C\left(1+\sup \left|\zeta_{t}\right|^{2}\right) \chi_{*}^{12} T M_{*}^{4}\left(M_{*}+1\right)^{8},
$$

and the sum of the last two terms on the right-hand side is bounded by

$$
C\left(1+\sup |\nabla \zeta|^{2}+\sup \left|\zeta_{t}\right|^{2}\right) \chi_{*}^{2(4+a)} M_{*}^{2}\left(M_{*}+1\right)^{4} \int_{0}^{T} \int_{U} \mathcal{K}|\nabla u|^{2} d x d t .
$$


Hence,

$$
\begin{aligned}
I \leq & C\left(1+\sup |\nabla \zeta|^{2}+\sup \left|\zeta_{t}\right|^{2}\right)\left\{\chi_{*}^{2} M_{*}^{2} \phi \mathcal{D}_{0}+\chi_{*}^{12} T M_{*}^{4}\left(M_{*}+1\right)^{8}\right. \\
& \left.+\chi_{*}^{2(4+a)} M_{*}^{2}\left(M_{*}+1\right)^{4} \int_{0}^{T} \int_{U} \mathcal{K}|\nabla u|^{2} d x d t\right\} .
\end{aligned}
$$

Estimating the last integral by (5.20) gives

$$
\begin{aligned}
I \leq & C\left(1+\sup |\nabla \zeta|^{2}+\sup \left|\zeta_{t}\right|^{2}\right)\left\{\chi_{*}^{2} M_{*}^{2} \phi \mathcal{D}_{0}+\chi_{*}^{12} T M_{*}^{4}\left(M_{*}+1\right)^{8}\right. \\
& \left.+\chi_{*}^{2(4+a)} M_{*}^{2}\left(M_{*}+1\right)^{4}\left[\chi_{*}^{2} \phi\left\|\bar{u}_{0}\right\|_{L^{2}}^{2}+\chi_{*}^{2(4+a)} T M_{*}^{2}\left(M_{*}+1\right)^{2}+\chi_{*}^{2(2+a)} \mathcal{E}_{*}\right]\right\} .
\end{aligned}
$$

Grouping the like-terms on the right-hand side and using simple estimations yield inequality (6.17).

By selecting the cut-off function $\zeta$ in (6.17) appropriately, we derive the spatial, as well as the spatial-temporal, interior estimates for $|\nabla u|$.

Notation. For simplicity, we will write $V \Subset U$ to indicate that $V$ is an open, relatively compact subset of $U$.

Theorem 6.4. Let $U^{\prime} \Subset U$.

(i) One has

$$
\begin{aligned}
\int_{0}^{T} \int_{U^{\prime}} \mathcal{K}|\nabla u|^{4} d x d t \leq & C\left\{\chi_{*}^{2(5+a)} M_{*}^{2}\left(M_{*}+1\right)^{4} \phi\left(\left\|\bar{u}_{0}\right\|_{L^{2}}^{2}+\left\|\nabla u_{0}\right\|_{L^{2}}^{2}\right)\right. \\
& \left.+\chi_{*}^{4(4+a)} T M_{*}^{4}\left(M_{*}+1\right)^{8}+\chi_{*}^{4(3+a)} M_{*}^{2}\left(M_{*}+1\right)^{4} \mathcal{E}_{*}\right\} .
\end{aligned}
$$

Consequently,

$$
\int_{0}^{T} \int_{U^{\prime}} \mathcal{K}|\nabla u|^{4} d x d t \leq C \chi_{*}^{4(4+a)} M_{*}^{2}\left(M_{*}+1\right)^{10} \mathcal{N}_{2}
$$

(ii) If $T_{0}$ is any number in $(0, T)$, then

$$
\begin{aligned}
\int_{T_{0}}^{T} \int_{U^{\prime}} \mathcal{K}|\nabla u|^{4} d x d t \leq & C\left(1+T_{0}^{-1}\right)^{2}\left\{\chi_{*}^{2(5+a)} M_{*}^{2}\left(M_{*}+1\right)^{4} \phi\left\|\bar{u}_{0}\right\|_{L^{2}}^{2}\right. \\
& \left.+\chi_{*}^{4(4+a)} T M_{*}^{4}\left(M_{*}+1\right)^{8}+\chi_{*}^{4(3+a)} M_{*}^{2}\left(M_{*}+1\right)^{4} \mathcal{E}_{*}\right\} .
\end{aligned}
$$

Consequently,

$$
\int_{T_{0}}^{T} \int_{U^{\prime}} \mathcal{K}|\nabla u|^{4} d x d t \leq C\left(1+T_{0}^{-1}\right)^{2} \chi_{*}^{4(4+a)} M_{*}^{2}\left(M_{*}+1\right)^{10} \mathcal{N}_{*} .
$$

(iii) If $s \in[2,4]$, then

$$
\int_{0}^{T} \int_{U^{\prime}} \mathcal{K}|\nabla u|^{s} d x d t \leq C \chi_{*}^{(4+a) s} M_{*}^{s-2}\left(M_{*}+1\right)^{3 s-2} \mathcal{N}_{2},
$$

and, for $T_{0} \in(0, T)$,

$$
\int_{T_{0}}^{T} \int_{U^{\prime}} \mathcal{K}|\nabla u|^{s} d x d t \leq C\left(1+T_{0}^{-1}\right)^{s-2} \chi_{*}^{(4+a) s} M_{*}^{s-2}\left(M_{*}+1\right)^{3 s-2} \mathcal{N}_{*} .
$$


Proof. (i) We fix a cut-off function $\zeta=\zeta(x)$ with $\zeta \equiv 1$ on $U^{\prime}$. We have $|\nabla \zeta| \leq C$ and $\zeta_{t} \equiv 0$. Then, by using inequality (6.17), we obtain

$$
\begin{aligned}
\int_{0}^{T} \int_{U^{\prime}} \mathcal{K}|\nabla u|^{4} d x d t \leq & \int_{0}^{T} \int_{U} \mathcal{K}|\nabla u|^{4} \zeta^{2} d x d t \\
\leq & C\left\{\chi_{*}^{2(5+a)} M_{*}^{2}\left(M_{*}+1\right)^{4} \phi\left(\left\|\bar{u}_{0}\right\|_{L^{2}}^{2}+\mathcal{D}_{0}\right)\right. \\
& \left.+\chi_{*}^{4(4+a)} T M_{*}^{4}\left(M_{*}+1\right)^{8}+\chi_{*}^{4(3+a)} M_{*}^{2}\left(M_{*}+1\right)^{4} \mathcal{E}_{*}\right\} .
\end{aligned}
$$

This proves (6.18). Now, note on the right-hand side of (6.18) that

$$
\chi_{*}^{2(5+a)} M_{*}^{2}\left(M_{*}+1\right)^{4}, \chi_{*}^{4(4+a)} M_{*}^{4}\left(M_{*}+1\right)^{8}, \chi_{*}^{4(3+a)} M_{*}^{2}\left(M_{*}+1\right)^{4} \leq \chi_{*}^{4(4+a)} M_{*}^{2}\left(M_{*}+1\right)^{10} .
$$

Utilizing these estimates, we obtain (6.19) from (6.18).

(ii) We select a different cut-off function $\zeta=\zeta(x, t)$ such that $\zeta=0$ for $0 \leq t \leq T_{0} / 2$, and $\zeta=1$ on $U^{\prime} \times\left[T_{0}, T\right]$, and its derivatives satisfy

$$
|\nabla \zeta| \leq C \text { and } 0 \leq \zeta_{t} \leq C T_{0}^{-1}
$$

where $C>0$ is independent of $T_{0}, T$.

With this function $\zeta$, it is obvious from (6.1) that $\mathcal{D}_{0}=0$. Then, by (6.17), we have

$$
\begin{aligned}
\int_{T_{0}}^{T} \int_{U^{\prime}} \mathcal{K}|\nabla u|^{4} d x d t \leq & \int_{0}^{T} \int_{U} \mathcal{K}|\nabla u|^{4} \zeta^{2} d x d t \\
\leq & C\left(1+T_{0}^{-2}\right)\left\{C \chi_{*}^{2(5+a)} M_{*}^{2}\left(M_{*}+1\right)^{4} \phi\left\|\bar{u}_{0}\right\|_{L^{2}}^{2}\right. \\
& \left.+\chi_{*}^{4(4+a)} T M_{*}^{4}\left(M_{*}+1\right)^{8}+\chi_{*}^{4(3+a)} M_{*}^{2}\left(M_{*}+1\right)^{4} \mathcal{E}_{*}\right\},
\end{aligned}
$$

which gives (6.20). Utilizing (6.24) again for the right-hand side of (6.20), we obtain (6.21).

(iii) The inequalities (6.22) and (6.23) already hold for $s=2$ thanks to (5.23) and (5.19), and for $s=4$ thanks to (6.18) and (6.19).

Consider $2<s<4$ now. By interpolation inequality (2.1), we have

$$
\int_{0}^{T} \int_{U^{\prime}} \mathcal{K}|\nabla u|^{s} d x d t \leq\left(\int_{0}^{T} \int_{U^{\prime}} \mathcal{K}|\nabla u|^{2} d x d t\right)^{\frac{4-s}{2}}\left(\int_{0}^{T} \int_{U^{\prime}} \mathcal{K}|\nabla u|^{4} d x d t\right)^{\frac{s-2}{2}} .
$$

Applying inequality (5.23), respectively (6.19), to estimate the first, respectively second, integral on the right-hand side, we obtain

$$
\int_{0}^{T} \int_{U^{\prime}} \mathcal{K}|\nabla u|^{s} d x d t \leq C\left(\chi_{*}^{2(4+a)}\left(M_{*}+1\right)^{4} \mathcal{N}_{*}\right)^{\frac{4-s}{2}}\left(\chi_{*}^{4(4+a)} M_{*}^{2}\left(M_{*}+1\right)^{10} \mathcal{N}_{2}\right)^{\frac{s-2}{2}},
$$

which yields (6.22).

Similarly, by (2.1), (5.23) and (6.21), we have

$$
\begin{aligned}
\int_{T_{0}}^{T} \int_{U^{\prime}} \mathcal{K}|\nabla u|^{s} d x d t & \leq\left(\int_{T_{0}}^{T} \int_{U^{\prime}} \mathcal{K}|\nabla u|^{2} d x d t\right)^{\frac{4-s}{2}}\left(\int_{T_{0}}^{T} \int_{U^{\prime}} \mathcal{K}|\nabla u|^{4} d x d t\right)^{\frac{s-2}{2}} \\
& \leq C\left(\chi_{*}^{2(4+a)}\left(M_{*}+1\right)^{4} \mathcal{N}_{*}\right)^{\frac{4-s}{2}}\left(\left(1+T_{0}^{-1}\right)^{2} \chi_{*}^{4(4+a)} M_{*}^{2}\left(M_{*}+1\right)^{10} \mathcal{N}_{*}\right)^{\frac{s-2}{2}}
\end{aligned}
$$

which implies (6.23). 
The estimates obtained in Theorem 6.4 contain the weight $\mathcal{K}(x, t)$. Below, we derive the estimates for the standard Lebesgue $L_{x, t}^{4-a}$-norm (without that weight).

Corollary 6.5. Let $U^{\prime} \Subset U$ and $T_{0} \in(0, T)$.

(i) One has

$$
\int_{0}^{T} \int_{U^{\prime}}|\nabla u|^{4-a} d x d t \leq C \chi_{*}^{4(4+a)}\left(M_{*}+1\right)^{12} \mathcal{N}_{2}
$$

and

$$
\int_{T_{0}}^{T} \int_{U^{\prime}}|\nabla u|^{4-a} d x d t \leq C\left(1+T_{0}^{-1}\right)^{2} \chi_{*}^{4(4+a)}\left(M_{*}+1\right)^{12} \mathcal{N}_{*} .
$$

(ii) If $s$ is any number in $(2-a, 4-a)$, then

$$
\int_{0}^{T} \int_{U^{\prime}}|\nabla u|^{s} d x d t \leq C \chi_{*}^{(4+a)(s+a)}\left(M_{*}+1\right)^{4(s+a-1)} \mathcal{N}_{2},
$$

and

$$
\int_{T_{0}}^{T} \int_{U^{\prime}}|\nabla u|^{s} d x d t \leq C\left(1+T_{0}^{-1}\right)^{s+a-2} \chi_{*}^{(4+a)(s+a)}\left(M_{*}+1\right)^{4(s+a-1)} \mathcal{N}_{*} .
$$

Proof. (i) Applying (4.6) to $s=4-a$ gives

$$
|\nabla u|^{4-a} \leq C \mathcal{K}|\nabla u|^{4}+C\left(1+M_{*} \chi_{*}\right)^{8} .
$$

Combining this with (6.19), respectively (6.21), we obtain (6.26), respectively (6.27).

(ii) Consider $2-a<s<4-a$. By interpolation inequality (2.1) and then using (5.24), (5.19) and (6.26), we have

$$
\begin{aligned}
& \int_{0}^{T} \int_{U^{\prime}}|\nabla u|^{s} d x d t \leq\left(\int_{0}^{T} \int_{U^{\prime}}|\nabla u|^{2-a} d x d t\right)^{\frac{4-a-s}{2}}\left(\int_{0}^{T} \int_{U^{\prime}}|\nabla u|^{4-a} d x d t\right)^{\frac{s-(2-a)}{2}} \\
& \leq C\left[\chi_{*}^{2(4+a)}\left(M_{*}+1\right)^{4} \mathcal{N}_{*}\right]^{\frac{4-a-s}{2}}\left[\chi_{*}^{4(4+a)}\left(M_{*}+1\right)^{12} \mathcal{N}_{2}\right]^{\frac{s-(2-a)}{2}} \\
& \leq C \chi_{*}^{(4+a)(s+a)}\left(M_{*}+1\right)^{4(s+a-1)} \mathcal{N}_{2} .
\end{aligned}
$$

Thus, we obtain (6.28).

Similarly, by (2.1), (5.24) and (6.27) we have

$$
\begin{aligned}
& \int_{T_{0}}^{T} \int_{U^{\prime}}|\nabla u|^{s} d x d t \leq\left(\int_{T_{0}}^{T} \int_{U^{\prime}}|\nabla u|^{2-a} d x d t\right)^{\frac{4-a-s}{2}}\left(\int_{T_{0}}^{T} \int_{U^{\prime}}|\nabla u|^{4-a} d x d t\right)^{\frac{s-(2-a)}{2}} \\
& \leq C\left[\chi_{*}^{2(4+a)}\left(M_{*}+1\right)^{4} \mathcal{N}_{*}\right]^{\frac{4-a-s}{2}}\left[\left(1+T_{0}^{-1}\right)^{2} \chi_{*}^{4(4+a)}\left(M_{*}+1\right)^{12} \mathcal{N}_{*}\right]^{\frac{s-(2-a)}{2}} \\
& \leq C\left(1+T_{0}^{-1}\right)^{s+a-2} \chi_{*}^{(4+a)(s+a)}\left(M_{*}+1\right)^{4(s+a-1)} \mathcal{N}_{*} .
\end{aligned}
$$

Thus, we obtain (6.29).

Remark 6.6. The estimate (6.29) of the $L_{x, t}^{s}$-norm of $\nabla u(x, t)$, for $t>0$, requires, as far as the initial data $u_{0}$ is concerned, at most the $L^{\infty}$-norm of $u_{0}$. Therefore, it shows the (formal) regularization effect of the PDE (1.21). This observation also applies to Corollary 6.11 and Theorem 7.2 below. 


\subsection{Estimates for higher $L_{x, t}^{s}$-norms}

In this subsection, we have estimates for the $L_{x, t}^{s}$-norms of $\nabla u$ with $s>4-a$.

Lemma 6.7. Let $s>2$, and $V$ be an open subset of $U$.

(i) If $\zeta=\zeta(x)$ with compact support in $V$, then

$$
\begin{aligned}
\int_{0}^{T} \int_{U} \mathcal{K}|\nabla u|^{s+2} \zeta^{2} d x d t \leq C\left(1+\sup |\nabla \zeta|^{2}\right) \\
\cdot\left\{\chi_{*}^{4(s+2+a)} M_{*}^{2}\left(M_{*}+1\right)^{4 s+2} \mathcal{N}_{s}+\chi_{*}^{2(4+a)} M_{*}^{2}\left(M_{*}+1\right)^{4} \int_{0}^{T} \int_{V} \mathcal{K}|\nabla u|^{s} d x d t\right\} .
\end{aligned}
$$

(ii) If $\zeta=\zeta(x, t)$ with $\zeta(x, 0) \equiv 0$ and, for each $t \in[0, T]$, the mapping $\zeta(\cdot, t)$ has compact support in $V$, then

$$
\begin{aligned}
\int_{0}^{T} \int_{U} \mathcal{K}|\nabla u|^{s+2} \zeta^{2} d x d t \leq C\left(1+\sup |\nabla \zeta|^{2}+\sup \left|\zeta_{t}\right|^{2}\right) \\
\cdot\left\{\chi_{*}^{4(s+2+a)} M_{*}^{2}\left(M_{*}+1\right)^{4 s+2} \mathcal{N}_{*}+\chi_{*}^{2(4+a)} M_{*}^{2}\left(M_{*}+1\right)^{6} \int_{0}^{T} \int_{V} \mathcal{K}|\nabla u|^{s} d x d t\right\} .
\end{aligned}
$$

Proof. Denote

$$
I=\int_{0}^{T} \int_{U} \mathcal{K}|\nabla u|^{2(s+2)} \zeta^{2} d x d t \text { and } J=\int_{0}^{T} \int_{V} \mathcal{K}|\nabla u|^{2(s+1)} d x d t .
$$

(i) Consider $s>0$. We estimate $I$ by (6.13), neglecting the second term on the left-hand side. Note in this case that $\zeta_{t}=0$ and hence $J_{*}=0$. We then use (6.17) to estimate the last term of $I_{*}$. The result is

$$
\begin{aligned}
I \leq & C M_{*}^{2} \chi_{*}^{2} \phi \mathcal{D}_{s}+C T \chi_{*}^{4(2 s+3)} M_{*}^{6}\left(M_{*}+1\right)^{8 s+6}+C \chi_{*}^{2(4+a)} M_{*}^{2}\left(M_{*}+1\right)^{4}\left(1+\sup |\nabla \zeta|^{2}\right) J \\
& +C\left(1+\sup |\nabla \zeta|^{2}\right)\left[\chi_{*}^{2(5+a)} M_{*}^{2}\left(M_{*}+1\right)^{4} \phi\left(\left\|\bar{u}_{0}\right\|_{L^{2}}^{2}+\left\|\nabla u_{0}\right\|_{L^{2}}^{2}\right)+T \chi_{*}^{4(4+a)} M_{*}^{4}\left(M_{*}+1\right)^{8}\right. \\
& \left.+\chi_{*}^{4(3+a)} M_{*}^{2}\left(M_{*}+1\right)^{4} \mathcal{E}_{*}\right] .
\end{aligned}
$$

For the terms containing the initial data, we estimate

$$
M_{*}^{2} \chi_{*}^{2} \leq \chi_{*}^{2(5+a)} M_{*}^{2}\left(M_{*}+1\right)^{4},
$$

and for the terms containing $T$, we use

$$
\chi_{*}^{4(2 s+3)} M_{*}^{6}\left(M_{*}+1\right)^{8 s+6}, \chi_{*}^{4(4+a)} M_{*}^{4}\left(M_{*}+1\right)^{8} \leq \chi_{*}^{8(s+2)+4 a} M_{*}^{4}\left(M_{*}+1\right)^{8(s+1)} .
$$

Hence, we obtain

$$
\begin{aligned}
& I \leq C\left(1+\sup |\nabla \zeta|^{2}\right)\left\{\chi_{*}^{2(5+a)} M_{*}^{2}\left(M_{*}+1\right)^{4} \phi\left(\left\|\bar{u}_{0}\right\|_{L^{2}}^{2}+\left\|\nabla u_{0}\right\|_{L^{2}}^{2}+\left\|\nabla u_{0}\right\|_{L^{2 s+2}}^{2 s+2}\right)\right. \\
& \left.+T \chi_{*}^{8(s+2)+4 a} M_{*}^{4}\left(M_{*}+1\right)^{8(s+1)}+\chi_{*}^{4(3+a)} M_{*}^{2}\left(M_{*}+1\right)^{4} \mathcal{E}_{*}+\chi_{*}^{2(4+a)} M_{*}^{2}\left(M_{*}+1\right)^{4} J\right\} .
\end{aligned}
$$


Now, consider $s>2$. By replacing $2 s+2$ in (6.33) with $s$, noting that

$$
I \text { becomes } \int_{0}^{T} \int_{U} \mathcal{K}|\nabla u|^{s+2} \zeta^{2} d x d t, \quad J \text { becomes } \int_{0}^{T} \int_{V} \mathcal{K}|\nabla u|^{s} d x d t,
$$

the power $8(s+2)+4 a$ becomes $4(s+2+a)$, and the power $8(s+1)$ becomes $4 s$, we obtain

$$
\begin{aligned}
& \int_{0}^{T} \int_{U} \mathcal{K}|\nabla u|^{s+2} \zeta^{2} d x d t \leq C\left(1+\sup |\nabla \zeta|^{2}\right) \\
& \quad \cdot\left\{\chi_{*}^{2(5+a)} M_{*}^{2}\left(M_{*}+1\right)^{4} \phi\left(\left\|\bar{u}_{0}\right\|_{L^{2}}^{2}+\left\|\nabla u_{0}\right\|_{L^{2}}^{2}+\left\|\nabla u_{0}\right\|_{L^{s}}^{s}\right)+T \chi_{*}^{4(s+2+a)} M_{*}^{4}\left(M_{*}+1\right)^{4 s}\right. \\
& \left.\quad+\chi_{*}^{4(3+a)} M_{*}^{2}\left(M_{*}+1\right)^{4} \mathcal{E}_{*}+\chi_{*}^{2(4+a)} M_{*}^{2}\left(M_{*}+1\right)^{4} \int_{0}^{T} \int_{V} \mathcal{K}|\nabla u|^{s} d x d t\right\} . \quad(6
\end{aligned}
$$

On the right-hand side of (6.34), in order to group the terms $\phi\left(\left\|\bar{u}_{0}\right\|_{L^{2}}^{2}+\left\|\nabla u_{0}\right\|_{L^{2}}^{2}+\left\|\nabla u_{0}\right\|_{L^{s}}^{s}\right)$, $T, \mathcal{E}_{*}$ together, we estimate their coefficients by

$$
\chi_{*}^{2(5+a)} M_{*}^{2}\left(M_{*}+1\right)^{4}, \chi_{*}^{4(s+2+a)} M_{*}^{4}\left(M_{*}+1\right)^{4 s}, \chi_{*}^{4(3+a)} M_{*}^{2}\left(M_{*}+1\right)^{4} \leq \chi_{*}^{4(s+2+a)} M_{*}^{2}\left(M_{*}+1\right)^{4 s+2} .
$$

Then inequality (6.30) follows (6.34).

(ii) Consider $s>0$. Note that $\mathcal{D}_{0}=\mathcal{D}_{s}=0$. We have from (6.13) that

$$
\begin{aligned}
I \leq & C\left(1+\sup |\nabla \zeta|^{2}+\sup \left|\zeta_{t}\right|^{2}\right)\left\{T \cdot\left[\chi_{*}^{4(2 s+3)} M_{*}^{6}\left(M_{*}+1\right)^{8 s+6}+\chi_{*}^{4(1+a)} M_{*}^{4}\left(M_{*}+1\right)^{4 a}\right]\right. \\
& \left.+\left[\chi_{*}^{2(4+a)} M_{*}^{2}\left(M_{*}+1\right)^{4}+\chi_{*}^{4(1+a)} M_{*}^{4}\left(M_{*}+1\right)^{4 a}\right] \cdot J\right\}+M_{*}^{2} \int_{0}^{T} \int_{U} \mathcal{K}\left|D^{2} u\right|^{2} \zeta^{2} d x d t .
\end{aligned}
$$

We use (6.17) to estimate the last term $M_{*}^{2} \int_{0}^{T} \int_{U} \mathcal{K}\left|D^{2} u\right|^{2} \zeta^{2} d x d t$. For the $T$-term, we use (6.32) again. For the $J$-term we use

$$
\chi_{*}^{2(4+a)} M_{*}^{2}\left(M_{*}+1\right)^{4}, \chi_{*}^{4(1+a)} M_{*}^{4}\left(M_{*}+1\right)^{4 a} \leq \chi_{*}^{2(4+a)} M_{*}^{2}\left(M_{*}+1\right)^{6} .
$$

Combining these estimates gives

$$
\begin{aligned}
I \leq & C\left(1+\sup |\nabla \zeta|^{2}+\sup \left|\zeta_{t}\right|^{2}\right)\left\{T \chi_{*}^{8(s+2)+4 a} M_{*}^{4}\left(M_{*}+1\right)^{8(s+1)}+\chi_{*}^{2(4+a)} M_{*}^{2}\left(M_{*}+1\right)^{6} J\right. \\
& \left.+\chi_{*}^{2(5+a)} M_{*}^{2}\left(M_{*}+1\right)^{4} \phi\left\|\bar{u}_{0}\right\|_{L^{2}}^{2}+T \chi_{*}^{4(4+a)} M_{*}^{4}\left(M_{*}+1\right)^{8}+\chi_{*}^{4(3+a)} M_{*}^{2}\left(M_{*}+1\right)^{4} \mathcal{E}_{*}\right\} .
\end{aligned}
$$

Simplifying the right-hand side once more, we obtain

$$
\begin{aligned}
I \leq & C\left(1+\sup |\nabla \zeta|^{2}+\sup \left|\zeta_{t}\right|^{2}\right)\left\{\chi_{*}^{2(5+a)} M_{*}^{2}\left(M_{*}+1\right)^{4} \phi\left\|\bar{u}_{0}\right\|_{L^{2}}^{2}\right. \\
& +T \chi_{*}^{8(s+2)+4 a} M_{*}^{4}\left(M_{*}+1\right)^{8(s+1)}+\chi_{*}^{4(3+a)} M_{*}^{2}\left(M_{*}+1\right)^{4} \mathcal{E}_{*} \\
& \left.+\chi_{*}^{2(4+a)} M_{*}^{2}\left(M_{*}+1\right)^{6} J\right\} .
\end{aligned}
$$

Same as in the proof of part (i), when $s>2$, replacing $2 s+2$ in (6.35) with $s$ yields (6.31). 
Theorem 6.8. If $U^{\prime} \Subset U$ and $s \geq 4$, then

$$
\int_{0}^{T} \int_{U^{\prime}} \mathcal{K}|\nabla u|^{s} d x d t \leq C \chi_{*}^{(4+a)(s+2)} M_{*}^{2}\left(M_{*}+1\right)^{4 s} \mathcal{N}_{s-2}
$$

Proof. (a) When $s=4$ the inequality (6.36) holds true thanks to the estimate (6.19). Hence we only focus on the case $s>4$.

(b) Consider the case $s=s_{*}+2 m$ with $s_{*}>2$ and $m \in \mathbb{N}$. Let $V$ be an open subset of $U$ such that $U^{\prime} \Subset V \Subset U$. We claim that

$$
\begin{aligned}
\int_{0}^{T} \int_{U^{\prime}} \mathcal{K}|\nabla u|^{s} d x d t \leq & C \chi_{*}^{2(4+a) m} M_{*}^{2 m}\left(M_{*}+1\right)^{4 m} \int_{0}^{T} \int_{V} \mathcal{K}|\nabla u|^{s_{*}} d x d t \\
& +C \chi_{*}^{(4+a) s} M_{*}^{2}\left(M_{*}+1\right)^{4 s-6} \mathcal{N}_{s-2}
\end{aligned}
$$

Proof of (6.37). Let $\left\{U_{k}\right\}_{k=0}^{m}$ be a family of smooth, open subsets of $U$ such that

$$
U^{\prime} \subset U_{m} \Subset U_{m-1} \Subset U_{m-2} \Subset \ldots \Subset U_{1} \Subset U_{0} \subset V \Subset U
$$

Denote $y_{k}=\int_{0}^{T} \int_{U_{k}} \mathcal{K}|\nabla u|^{s_{*}+2 k} d x d t$ for $0 \leq k \leq m$.

Let $k \in\{0,1,2, \ldots, m-1\}$. Choose $\zeta=\zeta_{k}(x)$, a $C^{2}$ cut-off function which is equal to 1 on $U_{k+1}$ and has compact support in $U_{k}$. Applying (6.30) to $s:=s_{*}+2 k$, we have

$$
y_{k+1} \leq A y_{k}+B
$$

where $A=C_{k} \chi_{*}^{2(4+a)} M_{*}^{2}\left(M_{*}+1\right)^{4}$ and $B=C_{k} \chi_{*}^{4\left(s_{*}+2 k+2+a\right)} M_{*}^{2}\left(M_{*}+1\right)^{4\left(s_{*}+2 k\right)+2} \widehat{B}$, with

$$
\widehat{B}=\phi\left(\left\|\bar{u}_{0}\right\|_{L^{2}}^{2}+\left\|\nabla u_{0}\right\|_{L^{2}}^{2}+\left\|\nabla u_{0}\right\|_{L_{s_{*}+2 k}}^{s_{*}+2 k}\right)+T+\mathcal{E}_{*}
$$

for some $C_{k}>0$ independent of $T$. Note that

$$
\left\|\nabla u_{0}\right\|_{L_{s_{*}+2 k}}^{s_{*}+2 k}=\int_{U}\left|\nabla u_{0}\right|^{s_{*}+2 k} d x \leq \int_{U}\left[\left|\nabla u_{0}\right|^{2}+\left|\nabla u_{0}\right|^{s_{*}+2(m-1)}\right] d x .
$$

Hence,

$$
\widehat{B} \leq \phi\left(\left\|\bar{u}_{0}\right\|_{L^{2}}^{2}+2\left\|\nabla u_{0}\right\|_{L^{2}}^{2}+\left\|\nabla u_{0}\right\|_{L^{s_{*}+2(m-1)}}^{s_{*}+2(m-1)}\right)+T+\mathcal{E}_{*} \leq 2 \mathcal{N}_{s-2} .
$$

Let $C_{*}=2 \max \left\{C_{k}: k=1,2, \ldots, m-1\right\}$. Hence,

$$
y_{k+1} \leq A_{*} y_{k}+B_{k}
$$

where

$$
A_{*}=C_{*} \chi_{*}^{2(4+a)} M_{*}^{2}\left(M_{*}+1\right)^{4}, \quad B_{k}=C_{*} \chi_{*}^{4\left(s_{*}+2 k+2+a\right)} M_{*}^{2}\left(M_{*}+1\right)^{4\left(s_{*}+2 k\right)+2} \mathcal{N}_{s-2}=B_{*} S^{k},
$$

with $S=\chi_{*}^{8}\left(M_{*}+1\right)^{8}$ and $B_{*}=A_{*} \chi_{*}^{4 s_{*}+2 a}\left(M_{*}+1\right)^{4 s_{*}-2} \mathcal{N}_{s-2}$. 
Iterating (6.40), we obtain

$$
\begin{aligned}
y_{k+1} & \leq A_{*}\left(A_{*} y_{k-1}+B_{k-1}\right)+B_{k}=A_{*}^{2} y_{k-1}+A_{*} B_{k-1}+B_{k} \\
& \leq A_{*}^{3} y_{k-2}+A_{*}^{2} B_{k-2}+A_{*} B_{k-1}+B_{k} \\
& \leq \cdots \leq A_{*}^{k+1} y_{0}+\sum_{j=0}^{k-1} A_{*}^{k-j} B_{j}+B_{k} .
\end{aligned}
$$

Letting $k=m-1$, we then have

$$
y_{m} \leq A_{*}^{m} y_{0}+\sum_{j=0}^{m-2} A_{*}^{m-1-j} B_{j}+B_{m-1} .
$$

Dealing with the middle sum on the right-hand side of (6.41), elementary calculations show, for $0 \leq j \leq m-2$, that

$$
\begin{aligned}
A_{*}^{m-j-1} B_{j} & =A_{*}^{m-j} \chi_{*}^{4 s_{*}+2 a+8 j}\left(M_{*}+1\right)^{4 s_{*}-2+8 j} \mathcal{N}_{s-2} \\
& =C \chi_{*}^{4\left(s_{*}+2 m\right)+2 a(m-j+1)} M_{*}^{2(m-j)}\left(M_{*}+1\right)^{4\left(s_{*}+2 m\right)-4(m-j)-2} \mathcal{N}_{s-2} \\
& \leq C \chi_{*}^{4 s+2 a(m+1)} \cdot\left[M_{*}^{2}\left(M_{*}+1\right)^{2(m-j-1)}\right] \cdot\left(M_{*}+1\right)^{4 s-4(m-j)-2} \mathcal{N}_{s-2} \\
& =C \chi_{*}^{4 s+2 a(m+1)} M_{*}^{2}\left(M_{*}+1\right)^{4 s-2(m-j)-4} \mathcal{N}_{s-2} .
\end{aligned}
$$

Note that $m-j \geq 2$ and

$$
s>2+2 m \text {. }
$$

Then we have

$$
A_{*}^{m-1-j} B_{j} \leq C \chi_{*}^{(4+a) s} M_{*}^{2}\left(M_{*}+1\right)^{4 s-8} \mathcal{N}_{s-2} .
$$

For the last term in (6.41), one has

$$
\begin{aligned}
B_{m-1} & =C \chi_{*}^{4\left(s_{*}+2 m+a\right)} M_{*}^{2}\left(M_{*}+1\right)^{4\left(s_{*}+2 m\right)-6} \mathcal{N}_{s-2} \\
& =C \chi_{*}^{4(s+a)} M_{*}^{2}\left(M_{*}+1\right)^{4 s-6} \mathcal{N}_{s-2} .
\end{aligned}
$$

Then combining (6.41) with (6.43) and (6.44) gives

$$
\begin{aligned}
y_{m} \leq & C \chi_{*}^{2(4+a) m} M_{*}^{2 m}\left(M_{*}+1\right)^{4 m} y_{0}+C(m-1) \chi_{*}^{(4+a) s} M_{*}^{2}\left(M_{*}+1\right)^{4 s-8} \mathcal{N}_{s-2} \\
& +C \chi_{*}^{4(s+a)} M_{*}^{2}\left(M_{*}+1\right)^{4 s-6} \mathcal{N}_{s-2} \\
\leq & C \chi_{*}^{2(4+a) m} M_{*}^{2 m}\left(M_{*}+1\right)^{4 m} y_{0}+C \chi_{*}^{(4+a) s} M_{*}^{2}\left(M_{*}+1\right)^{4 s-6} \mathcal{N}_{s-2} .
\end{aligned}
$$

Therefore, estimate (6.37) follows.

(c) Consider the general case $s>4$ now. Then there exist $s_{*} \in(2,4]$ and integer $m \geq 1$ such that $s=s_{*}+2 m$. We apply estimate (6.37) using the relation (6.42), and have

$$
\begin{aligned}
\int_{0}^{T} \int_{U^{\prime}} \mathcal{K}|\nabla u|^{s} d x d t \leq & C \chi_{*}^{(4+a)(s-2)} M_{*}^{2 m}\left(M_{*}+1\right)^{2(s-2)} \int_{0}^{T} \int_{V} \mathcal{K}|\nabla u|^{s_{*}} d x d t \\
& +C \chi_{*}^{(4+a) s} M_{*}^{2}\left(M_{*}+1\right)^{4 s-6} \mathcal{N}_{s-2} .
\end{aligned}
$$


Using (6.42) again,

$$
M_{*}^{2 m} \leq M_{*}^{2}\left(M_{*}+1\right)^{2 m-2} \leq M_{*}^{2}\left(M_{*}+1\right)^{s-4} .
$$

Then

$$
\begin{aligned}
\int_{0}^{T} \int_{U^{\prime}} \mathcal{K}|\nabla u|^{s} d x d t \leq & C \chi_{*}^{(4+a)(s-2)} M_{*}^{2}\left(M_{*}+1\right)^{3 s-8} \int_{0}^{T} \int_{V} \mathcal{K}|\nabla u|^{s_{*}} d x d t \\
& +C \chi_{*}^{(4+a) s} M_{*}^{2}\left(M_{*}+1\right)^{4 s-6} \mathcal{N}_{s-2}
\end{aligned}
$$

Note, by Young's inequality and applying (6.19) to $U^{\prime}:=V$, that

$$
\begin{aligned}
& \int_{0}^{T} \int_{V} \mathcal{K}|\nabla u|^{s_{*}} d x d t \leq C\left(T+\int_{0}^{T} \int_{V} \mathcal{K}|\nabla u|^{4} d x d t\right) \\
& \leq C \chi_{*}^{4(4+a)}\left(M_{*}+1\right)^{12}\left[\phi\left(\left\|\bar{u}_{0}\right\|_{L^{2}}^{2}+\left\|\nabla u_{0}\right\|_{L^{2}}^{2}\right)+T+\mathcal{E}_{*}\right] .
\end{aligned}
$$

Due to (5.19) we can conclude that

$$
\int_{0}^{T} \int_{U^{\prime}} \mathcal{K}|\nabla u|^{s} d x d t \leq C \chi_{*}^{(4+a)(s+2)} M_{*}^{2}\left(M_{*}+1\right)^{3 s+4} \mathcal{N}_{2}+C \chi_{*}^{(4+a) s} M_{*}^{2}\left(M_{*}+1\right)^{4 s-6} \mathcal{N}_{s-2},
$$

and we obtain (6.36).

Proposition 6.9. If $U^{\prime} \Subset V \Subset U$, and $s=s_{*}+2 m$ with $s_{*}>2$ and $m \in \mathbb{N}$, then

$$
\begin{aligned}
\int_{T_{0}}^{T} \int_{U^{\prime}} \mathcal{K}|\nabla u|^{s} d x d t \leq & C\left(1+t_{0}^{-1}\right)^{2 m} \chi_{*}^{2(4+a) m} M_{*}^{2 m}\left(M_{*}+1\right)^{6 m} \int_{T_{0}-t_{0}}^{T} \int_{V} \mathcal{K}|\nabla u|^{s_{*}} d x d t \\
& +C\left(1+t_{0}^{-1}\right)^{2 m} \chi_{*}^{(4+a) s} M_{*}^{2}\left(M_{*}+1\right)^{4 s-6} \mathcal{N}_{*} .
\end{aligned}
$$

for any numbers $T_{0}$ and $t_{0}$ such that $0<t_{0}<T_{0}<T$.

Proof. Let $\left\{U_{k}\right\}_{k=0}^{m}$ be as in (6.38). Let $\tau_{0}=T_{0}-t_{0}<\tau_{1}<\tau_{2}<\ldots<\tau_{m}=T_{0}$ be evenly paced. Define $y_{k}=\int_{\tau_{k}}^{T} \int_{U_{k}} \mathcal{K}|\nabla u|^{s_{*}+2 k} d x d t$ for $0 \leq k \leq m$.

Given $k \in\{0,1,2, \ldots, m-1\}$. Let $\zeta_{k}(x, t)$ be a smooth cut-off function which is equal to one on $U_{k+1} \times\left[\tau_{k+1}, T\right]$, has compact support in $U_{k} \times\left[\tau_{k}, T\right]$, and satisfies

$$
\left|\nabla \zeta_{k}\right| \leq C_{k}^{\prime}, \quad 0 \leq \zeta_{k, t} \leq \frac{2}{\tau_{k+1}-\tau_{k}}=\frac{2}{m t_{0}}
$$

where $C_{k}^{\prime}>0$ is independent of $T, T_{0}, t_{0}$. Then using $s:=s_{*}+2 k$ and $\zeta=\zeta_{k}$ in (6.31), we have the same relation (6.39), with the constants defined by

$$
\begin{aligned}
& A=C_{k}\left(1+t_{0}^{-2}\right) \chi_{*}^{2(4+a)} M_{*}^{2}\left(M_{*}+1\right)^{6}, \\
& B=C_{k}\left(1+t_{0}^{-2}\right) \chi_{*}^{4\left(s_{*}+2 k+2+a\right)} M_{*}^{2}\left(M_{*}+1\right)^{4\left(s_{*}+2 k\right)+2} \mathcal{N}_{*},
\end{aligned}
$$

for some positive constant $C_{k}$ independent of $T, T_{0}, t_{0}$. 
Set $C_{*}=\max \left\{C_{k}: k=0,1, \ldots, m-1\right\}$, we obtain (6.40) where

$$
\begin{aligned}
& A_{*}=C_{*}\left(1+t_{0}^{-1}\right)^{2} \chi_{*}^{2(4+a)} M_{*}^{2}\left(M_{*}+1\right)^{6}, \\
& B_{k}=C_{*}\left(1+t_{0}^{-1}\right)^{2} \chi_{*}^{4\left(s_{*}+2 k+2+a\right)} M_{*}^{2}\left(M_{*}+1\right)^{4\left(s_{*}+2 k\right)+2} \mathcal{N}_{*}=B_{*} S^{k}
\end{aligned}
$$

with the same $S=\chi_{*}^{8}\left(M_{*}+1\right)^{8}$, but

$$
B_{*}=C_{*}\left(1+t_{0}^{-1}\right)^{2} \chi_{*}^{4\left(s_{*}+2+a\right)} M_{*}^{2}\left(M_{*}+1\right)^{4 s_{*}+2} \mathcal{N}_{*}=A_{*} \chi_{*}^{4 s_{*}+2 a}\left(M_{*}+1\right)^{4 s_{*}-4} \mathcal{N}_{*} .
$$

Then we obtain (6.41) by iteration again. For $0 \leq j \leq m-2$,

$$
\begin{aligned}
A_{*}^{m-j-1} B_{j} & =A_{*}^{m-j} \chi_{*}^{4 s_{*}+2 a+8 j}\left(M_{*}+1\right)^{4 s_{*}+8 j-4} \mathcal{N}_{*} \\
& \leq C\left(1+t_{0}^{-1}\right)^{2(m-j)} \chi_{*}^{4\left(s_{*}+2 m\right)+2 a(m-j+1)} M_{*}^{2(m-j)}\left(M_{*}+1\right)^{4 s_{*}+6 m+2 j-4} \mathcal{N}_{*} .
\end{aligned}
$$

Simply estimating $M_{*}^{2(m-j)} \leq M_{*}^{2}\left(M_{*}+1\right)^{2(m-j-1)}$, we then have

$$
\begin{aligned}
A_{*}^{m-j-1} B_{j} & \leq C\left(1+t_{0}^{-1}\right)^{2(m-j)} \chi_{*}^{4\left(s_{*}+2 m\right)+2 a(m+1)} M_{*}^{2}\left(M_{*}+1\right)^{4 s_{*}+8 m-6} \mathcal{N}_{*} \\
& =C\left(1+t_{0}^{-1}\right)^{2 m} \chi_{*}^{(4+a) s} M_{*}^{2}\left(M_{*}+1\right)^{4 s-6} \mathcal{N}_{*} .
\end{aligned}
$$

Also,

$$
B_{m-1}=C\left(1+t_{0}^{-1}\right)^{2} \chi_{*}^{4(s+a)} M_{*}^{2}\left(M_{*}+1\right)^{4 s-6} \mathcal{N}_{*} .
$$

Thus, we have from (6.41) that

$$
\begin{aligned}
y_{m} \leq & C\left(1+t_{0}^{-1}\right)^{2 m} \chi_{*}^{2(4+a) m} M_{*}^{2 m}\left(M_{*}+1\right)^{6 m} y_{0} \\
& +C(m-1)\left(1+t_{0}^{-1}\right)^{2 m} \chi_{*}^{(4+a) s} M_{*}^{2}\left(M_{*}+1\right)^{4 s-6} \mathcal{N}_{*} \\
& +C\left(1+t_{0}^{-1}\right)^{2} \chi_{*}^{4(s+a)} M_{*}^{2}\left(M_{*}+1\right)^{4 s-6} \mathcal{N}_{*} .
\end{aligned}
$$

Hence, we obtain (6.45).

Theorem 6.10. If $U^{\prime} \Subset U$ and $s>4$, then one has, for any $T_{0} \in(0, T)$, that

$$
\int_{T_{0}}^{T} \int_{U^{\prime}} \mathcal{K}|\nabla u|^{s} d x d t \leq C\left(1+T_{0}^{-1}\right)^{s} \chi_{*}^{(4+a)(s+2)} M_{*}^{2}\left(M_{*}+1\right)^{4 s+2} \mathcal{N}_{*} .
$$

Proof. There exist $2<s_{*} \leq 4$ and integer $m \geq 1$ such that $s=s_{*}+2 m$. Let $t_{0}:=T_{0} / 2$, and $V$ be a set with $U^{\prime} \Subset V \Subset U$. Applying (6.45), we have

$$
\begin{aligned}
\int_{T_{0}}^{T} \int_{U^{\prime}} \mathcal{K}|\nabla u|^{s} d x d t \leq & C\left(1+T_{0}^{-1}\right)^{2 m} \chi_{*}^{2(4+a) m} M_{*}^{2 m}\left(M_{*}+1\right)^{6 m} y_{*} \\
& +C\left(1+T_{0}^{-1}\right)^{2 m} \chi_{*}^{(4+a) s} M_{*}^{2}\left(M_{*}+1\right)^{4 s-6} \mathcal{N}_{*} .
\end{aligned}
$$

where $y_{*}=\int_{T_{0} / 2}^{T} \int_{V} \mathcal{K}|\nabla u|^{s_{*}} d x d t$. By Young's inequality and (6.21) applied to $U^{\prime}:=V$, we have

$$
y_{*} \leq C\left(T+\int_{T_{0} / 2}^{T} \int_{V} \mathcal{K}|\nabla u|^{4} d x d t\right) \leq C\left(1+T_{0}^{-1}\right)^{2} \chi_{*}^{4(4+a)}\left(M_{*}+1\right)^{12} \mathcal{N}_{*} .
$$


Then

$$
\begin{aligned}
& C\left(1+T_{0}^{-1}\right)^{2 m} \chi_{*}^{2(4+a) m} M_{*}^{2 m}\left(M_{*}+1\right)^{6 m} y_{*} \leq C\left(1+T_{0}^{-1}\right)^{s-2} \chi_{*}^{(4+a)(s-2)} M_{*}^{2}\left(M_{*}+1\right)^{8 m-2} y_{*} \\
& \quad \leq C\left(1+T_{0}^{-1}\right)^{s} \chi_{*}^{(4+a)(s+2)} M_{*}^{2}\left(M_{*}+1\right)^{4 s+2} \mathcal{N}_{*} .
\end{aligned}
$$

Combining this with (6.47) gives (6.46).

Corollary 6.11. Let $U^{\prime} \Subset U$ and $s>4-a$. Then

$$
\int_{0}^{T} \int_{U^{\prime}}|\nabla u|^{s} d x d t \leq C \chi_{*}^{(4+a)(s+a+2)}\left(M_{*}+1\right)^{4(s+a+1 / 2)} \mathcal{N}_{s+a-2} .
$$

Moreover, it holds, for any number $T_{0} \in(0, T)$, that

$$
\int_{T_{0}}^{T} \int_{U^{\prime}}|\nabla u|^{s} d x d t \leq C\left(1+T_{0}^{-1}\right)^{s+a} \chi_{*}^{(4+a)(s+a+2)}\left(M_{*}+1\right)^{4(s+a+1)} \mathcal{N}_{*} .
$$

Proof. Using (4.6) and applying (6.36) with $s$ being substituted by $s+a$, we have

$$
\begin{aligned}
\int_{0}^{T} \int_{U^{\prime}}|\nabla u|^{s} d x d t & \leq C \int_{0}^{T} \int_{U^{\prime}}\left(\mathcal{K}|\nabla u|^{s+a}+\left(\chi_{*}\left(M_{*}+1\right)\right)^{2(s+a)}\right) d x d t \\
& \leq C \chi_{*}^{(4+a)(s+a+2)} M_{*}^{2}\left(M_{*}+1\right)^{4(s+a)} \mathcal{N}_{s+a-2}+C T \chi_{*}^{2(s+a)}\left(M_{*}+1\right)^{2(s+a)} .
\end{aligned}
$$

Note that $T \leq \mathcal{N}_{s+a-2}$. Then (6.48) follows. Similarly, using (6.46), instead of (6.36), we obtain (6.49).

\section{Gradient estimates (III)}

This section is focused on the estimates for the $L_{t}^{\infty} L_{x}^{s}$-norms of $\nabla u$. For $s \geq 2$, replacing $s$ in (6.3) with $s / 2-1$ gives

$$
\begin{aligned}
I \stackrel{\text { def }}{=} \phi \sup _{t \in[0, T]} \int_{U}|\nabla u(x, t)|^{s} \zeta^{2}(x, t) d x \\
\leq \phi \int_{U}\left|\nabla u_{0}(x)\right|^{s} \zeta^{2}(x, 0) d x+C \chi_{*}^{2(3+a)} M_{*}^{4} \int_{0}^{T} \int_{U} \mathcal{K}|\nabla u|^{s-2} \zeta^{2} d x d t \\
\quad+C \chi_{*}^{2(3+a)}\left(M_{*}+1\right)^{2} \int_{0}^{T} \int_{U} \mathcal{K}|\nabla u|^{s}\left(\zeta^{2}+|\nabla \zeta|^{2}\right) d x d t+C \int_{0}^{T} \int_{U}|\nabla u|^{s} \zeta\left|\zeta_{t}\right| d x d t .
\end{aligned}
$$

Theorem 7.1. If $U^{\prime} \Subset U$, then one has, for all $t \in[0, T]$, that

$$
\begin{aligned}
\phi \int_{U^{\prime}}|\nabla u(x, t)|^{s} d x \leq & \phi \int_{U}\left|\nabla u_{0}(x)\right|^{s} d x \\
& +C \begin{cases}\chi_{*}^{4(4+a)}\left(M_{*}+1\right)^{6} \mathcal{N}_{0} & \text { if } s=2, \\
\chi_{*}^{(s+2)(4+a)} M_{*}^{s-2}\left(M_{*}+1\right)^{3 s+2} \mathcal{N}_{2} & \text { if } 2<s \leq 4, \\
\chi_{*}^{(s+4)(4+a)} M_{*}^{2}\left(M_{*}+1\right)^{4(s+1)} \mathcal{N}_{s-2} & \text { if } s>4 .\end{cases}
\end{aligned}
$$


Proof. Denote $J=\phi \sup _{t \in[0, T]} \int_{U^{\prime}}|\nabla u(x, t)|^{s} d x$. Choose $\zeta$ to be the same function $\zeta(x)$ as in the proof of Theorem 6.4(i). Then we have the relation

$$
J \leq I .
$$

We then bound $I$ by using inequality (7.1), noticing that the last integral of this inequality vanishes, and the integrand of the second term on its right-hand side can be bounded by

$$
\mathcal{K}|\nabla u|^{s-2} \leq \mathcal{K}\left(1+|\nabla u|^{s}\right) \leq 1+\mathcal{K}|\nabla u|^{s} .
$$

After this, combining the two constants for the integrals involving $\mathcal{K}|\nabla u|^{s}$, we obtain

$$
\begin{aligned}
J \leq & \phi \int_{U}\left|\nabla u_{0}(x)\right|^{s} d x+C \chi_{*}^{2(3+a)} M_{*}^{4} T \\
& +C \chi_{*}^{2(3+a)}\left(M_{*}+1\right)^{4} \int_{0}^{T} \int_{U} \mathcal{K}|\nabla u|^{s}\left(\zeta^{2}+|\nabla \zeta|^{2}\right) d x d t .
\end{aligned}
$$

Consider $s=2$. Using (5.22) to estimate the last integral in (7.4), we obtain

$$
\begin{aligned}
J \leq & \phi \int_{U}\left|\nabla u_{0}(x)\right|^{s} d x+C \chi_{*}^{2(3+a)} M_{*}^{4} T \\
& +C \chi_{*}^{2(3+a)}\left(M_{*}+1\right)^{4} \cdot \chi_{*}^{2(4+a)}\left(M_{*}+1\right)^{2} \mathcal{N}_{0} .
\end{aligned}
$$

Making a generous bound $2(3+a)<2(4+a)$ for the first two exponents of $\chi_{*}$ above, we obtain the first estimate in (7.2).

Consider $2<s \leq 4$. Using (6.22) to estimate the last integral in (17.4), we obtain

$$
\begin{aligned}
J \leq & \phi \int_{U}\left|\nabla u_{0}(x)\right|^{s} d x+C \chi_{*}^{2(3+a)} M_{*}^{4} T \\
& +C \chi_{*}^{2(3+a)}\left(M_{*}+1\right)^{4} \cdot \chi_{*}^{(4+a) s} M_{*}^{s-2}\left(M_{*}+1\right)^{3 s-2} \mathcal{N}_{2} .
\end{aligned}
$$

Then the second estimate in (7.2) follows.

Consider $s>4$. Using (6.36) to estimate the last integral in (7.4), we have

$$
\begin{aligned}
J \leq & \phi \int_{U}\left|\nabla u_{0}(x)\right|^{s} d x+C \chi_{*}^{2(3+a)} M_{*}^{4} T \\
& +C \chi_{*}^{2(3+a)}\left(M_{*}+1\right)^{4} \cdot \chi_{*}^{(4+a)(s+2)} M_{*}^{2}\left(M_{*}+1\right)^{4 s} \mathcal{N}_{s-2} .
\end{aligned}
$$

With simple manipulations, we obtain from this the third estimate in (7.2).

Theorem 7.2. Let $U^{\prime} \Subset U$ and $T_{0} \in(0, T)$. Then it holds, for all $t \in\left[T_{0}, T\right]$, that

$$
\begin{aligned}
& \phi \int_{U^{\prime}}|\nabla u(x, t)|^{s} d x \leq C \\
& \cdot \begin{cases}\chi_{*}^{(4+a)^{2}}\left(1+T_{0}^{-1}\right)^{1+a}\left(M_{*}+1\right)^{2(3+a)}\left\{M_{*}^{a}\left(M_{*}+1\right)^{2+a} \mathcal{N}_{*}+\mathcal{N}_{0}\right\} & \text { if } s=2, \\
\chi_{*}^{(4+a)(s+a+2)}\left(1+T_{0}^{-1}\right)^{s+a-1} M_{*}^{s-2}\left(M_{*}+1\right)^{3 s+4 a+2} \mathcal{N}_{*} & \text { if } 2<s \leq 4-a, \\
\chi_{*}^{(4+a)(s+a+4)}\left(1+T_{0}^{-1}\right)^{s+a+1} M_{*}^{s-2}\left(M_{*}+1\right)^{3 s+4 a+10} \mathcal{N}_{*} & \text { if } 4-a<s \leq 4, \\
\chi_{*}^{(4+a)(s+a+4)}\left(1+T_{0}^{-1}\right)^{s+a+1} M_{*}^{2}\left(M_{*}+1\right)^{4 s+4 a+6} \mathcal{N}_{*} & \text { if } s>4 .\end{cases}
\end{aligned}
$$


Consequently, one has, for all $s \geq 2$ and $t \in\left[T_{0}, T\right]$, that

$$
\phi \int_{U^{\prime}}|\nabla u(x, t)|^{s} d x \leq C \chi_{*}^{(4+a)(s+a+4)}\left(1+T_{0}^{-1}\right)^{s+a+1}\left(M_{*}+1\right)^{4(s+a+2)} \mathcal{N}_{*} .
$$

Proof. Choose $\zeta(x, t)$ to be the cut-off function in the proof of Theorem 6.4(ii) which satisfies additionally that $\zeta$ has compact support in $V \times\left[T_{0} / 2, T\right]$, where $U^{\prime} \Subset V \Subset U$.

Let $J$ be the same as in Theorem [7.1. Again, we have (7.3), and use (7.1) to estimate $I$. Note, on the right-hand side of (7.1), that

$$
\mathcal{K}|\nabla u|^{s-2} \leq 1+|\nabla u|^{s}, \quad \mathcal{K}|\nabla u|^{s} \leq|\nabla u|^{s} .
$$

Utilizing these properties as well as (6.25), we have from (17.3) and (7.1) that

$$
J \leq C \chi_{*}^{2(3+a)} M_{*}^{4} T+C \chi_{*}^{2(3+a)}\left(M_{*}+1\right)^{4}\left(1+T_{0}^{-1}\right) \int_{T_{0} / 2}^{T} \int_{V}|\nabla u|^{s} d x d t .
$$

Estimate the last integral in (7.7),

$$
\begin{aligned}
& \int_{T_{0} / 2}^{T} \int_{V}|\nabla u|^{s} d x d t=\int_{T_{0} / 2}^{T} \int_{V} \mathcal{K}|\nabla u|^{s} \mathcal{K}^{-1} d x d t \\
& \leq C \int_{T_{0} / 2}^{T} \int_{V} \mathcal{K}\left(|\nabla u|^{s+a}+|\nabla u|^{s} \chi_{*}^{2 a}\left(M_{*}+1\right)^{2 a}\right) d x d t \\
& =C \int_{T_{0} / 2}^{T} \int_{V} \mathcal{K}|\nabla u|^{s+a} d x d t+C \chi_{*}^{2 a}\left(M_{*}+1\right)^{2 a} \int_{T_{0} / 2}^{T} \int_{V} \mathcal{K}|\nabla u|^{s} d x d t .
\end{aligned}
$$

Denote by $I_{1}$ and $I_{2}$ the last two double integrals. We estimate them, in calculations below, by using inequalities (6.23) and (6.46) with $T_{0}:=T_{0} / 2$ and $U^{\prime}:=V$.

Case $s=2$. Applying (6.23) to $s:=2+a \in(2,4)$ to bound $I_{1}$, and applying (5.22) to $s:=2$ to bound $I_{2}$ give

$$
\begin{aligned}
J \leq & C \chi_{*}^{2(3+a)} M_{*}^{4} T+C \chi_{*}^{2(3+a)}\left(M_{*}+1\right)^{4}\left(1+T_{0}^{-1}\right) \\
& \cdot\left\{\left(1+T_{0}^{-1}\right)^{a} \chi_{*}^{(4+a)(2+a)} M_{*}^{a}\left(M_{*}+1\right)^{4+3 a} \mathcal{N}_{*}+\chi_{*}^{2 a}\left(M_{*}+1\right)^{2 a} \cdot \chi_{*}^{2(4+a)}\left(M_{*}+1\right)^{2} \mathcal{N}_{0}\right\} \\
\leq & C \chi_{*}^{2(3+a)} M_{*}^{4} T+C \chi_{*}^{(4+a)^{2}}\left(1+T_{0}^{-1}\right)^{1+a} M_{*}^{a}\left(M_{*}+1\right)^{8+3 a} \mathcal{N}_{*} \\
& +C \chi_{*}^{14+6 a}\left(1+T_{0}^{-1}\right)\left(M_{*}+1\right)^{6+2 a} \mathcal{N}_{0} .
\end{aligned}
$$

We obtain the first estimate in (7.5).

Case $2<s \leq 4-a$. Estimating $I_{1}$ by (6.23) applied to $s:=s+a$, and estimating $I_{2}$ by (6.23), we have

$$
\begin{aligned}
J \leq & C \chi_{*}^{2(3+a)} M_{*}^{4} T+C \chi_{*}^{2(3+a)}\left(M_{*}+1\right)^{4}\left(1+T_{0}^{-1}\right) \\
& \cdot\left\{\left(1+T_{0}^{-1}\right)^{s+a-2} \chi_{*}^{(4+a)(s+a)} M_{*}^{s+a-2}\left(M_{*}+1\right)^{3(s+a)-2} \mathcal{N}_{*}\right. \\
& \left.+\chi_{*}^{2 a}\left(M_{*}+1\right)^{2 a} \cdot\left(1+T_{0}^{-1}\right)^{s-2} \chi_{*}^{(4+a) s} M_{*}^{s-2}\left(M_{*}+1\right)^{3 s-2} \mathcal{N}_{*}\right\} \\
\leq & C \chi_{*}^{2(3+a)} M_{*}^{4} T+C \chi_{*}^{(4+a)(s+a+2)}\left(1+T_{0}^{-1}\right)^{s+a-1} M_{*}^{s-2}\left(M_{*}+1\right)^{3 s+4 a+2} \mathcal{N}_{*} .
\end{aligned}
$$


We obtain the second estimate in (7.5).

Case $4-a<s \leq 4$. Estimating $I_{1}$ by (6.46) applied to $s:=s+a$, and estimating $I_{2}$ by (6.23) yield

$$
\begin{aligned}
J \leq & C \chi_{*}^{2(3+a)} M_{*}^{4} T+C \chi_{*}^{2(3+a)}\left(M_{*}+1\right)^{4}\left(1+T_{0}^{-1}\right) \\
& \cdot\left\{\left(1+T_{0}^{-1}\right)^{s+a} \chi_{*}^{(4+a)(s+a+2)} M_{*}^{2}\left(M_{*}+1\right)^{4(s+a)+2} \mathcal{N}_{*}\right. \\
& \left.+\chi_{*}^{2 a}\left(M_{*}+1\right)^{2 a} \cdot\left(1+T_{0}^{-1}\right)^{s-2} \chi_{*}^{(4+a) s} M_{*}^{s-2}\left(M_{*}+1\right)^{3 s-2} \mathcal{N}_{*}\right\} \\
\leq & C \chi_{*}^{2(3+a)} M_{*}^{4} T+C \chi_{*}^{(4+a)(s+a+4)}\left(1+T_{0}^{-1}\right)^{s+a+1} M_{*}^{s-2}\left(M_{*}+1\right)^{3 s+4 a+10} \mathcal{N}_{*} .
\end{aligned}
$$

We obtain the third estimate in (7.5).

Case $s>4$. Estimating $I_{1}$ by (6.46) for $s:=s+a$, and estimating $I_{2}$ by (6.46) result in

$$
\begin{aligned}
J \leq & C \chi_{*}^{2(3+a)} M_{*}^{4} T+C \chi_{*}^{2(3+a)}\left(M_{*}+1\right)^{4}\left(1+T_{0}^{-1}\right) \\
& \cdot\left\{\left(1+T_{0}^{-1}\right)^{s+a} \chi_{*}^{(4+a)(s+a+2)} M_{*}^{2}\left(M_{*}+1\right)^{4(s+a)+2} \mathcal{N}_{*}\right. \\
& \left.+\chi_{*}^{2 a}\left(M_{*}+1\right)^{2 a} \cdot\left(1+T_{0}^{-1}\right)^{s} \chi_{*}^{(4+a)(s+2)} M_{*}^{2}\left(M_{*}+1\right)^{4 s+2} \mathcal{N}_{*}\right\} \\
\leq & C \chi_{*}^{2(3+a)} M_{*}^{4} T+C \chi_{*}^{(4+a)(s+a+4)}\left(1+T_{0}^{-1}\right)^{s+a+1} M_{*}^{2}\left(M_{*}+1\right)^{4 s+4 a+6} \mathcal{N}_{*} .
\end{aligned}
$$

We obtain the fourth estimate in (7.5).

Finally, one can easily unify the estimates in (7.5) for all $s>2$ with (7.6)). This can also be done for the case $s=2$ by comparing $\mathcal{N}_{0}$ with $\mathcal{N}_{*}$ using the last relation in (5.18).

Remark 7.3. Similar to Remark 5.5, when $u, u_{0}, \bar{u}_{0}$ are small in necessary norms, and $\mathcal{E}_{*}$ is small, then $M_{*}$ and $\mathcal{N}_{0}$ are small, which make the the right-hand sides of (7.2) and (7.5) to be small.

Acknowledgments. The authors would like to thank Dat Cao, Akif Ibragimov and Tuoc Phan for very helpful discussions.

Data availability. The data that support the findings of this study are available from the corresponding author upon reasonable request. The data that supports the findings of this study are available within the article.

\section{References}

[1] Tarek Ahmed. Reservoir engineering handbook. Gulf Professional Publishing, 2nd edition, 2001.

[2] Eugenio Aulisa, Lidia Bloshanskaya, Luan Hoang, and Akif Ibragimov. Analysis of generalized Forchheimer flows of compressible fluids in porous media. J. Math. Phys., 50(10):103102, 44 pp, 2009.

[3] Jacob Bear. Dynamics of Fluids in Porous Media. Dover Publications, 1988. Reprint of the American Elsevier Publishing Company, Inc., New York, 1972 edition. 
[4] Miroslav Bulíček, Josef Málek, and Josef Žabenský. A generalization of the DarcyForchheimer equation involving an implicit, pressure-dependent relation between the drag force and the velocity. J. Math. Anal. Appl., 424(1):785-801, 2015.

[5] A. O. Çelebi, V. K. Kalantarov, and D. Uğurlu. On continuous dependence on coefficients of the Brinkman-Forchheimer equations. Appl. Math. Lett., 19(8):801-807, 2006.

[6] Emine Celik and Luan Hoang. Generalized Forchheimer flows in heterogeneous porous media. Nonlinearity, 29(3):1124-1155, 2016.

[7] Emine Celik and Luan Hoang. Maximum estimates for generalized Forchheimer flows in heterogeneous porous media. J. Differential Equations, 262(3):2158-2195, 2017.

[8] Emine Celik, Luan Hoang, Akif Ibragimov, and Thinh Kieu. Fluid flows of mixed regimes in porous media. J. Math. Phys., 58(2):023102, 30 pp, 2017.

[9] Emine Celik, Luan Hoang, and Thinh Kieu. Doubly nonlinear parabolic equations for a general class of Forchheimer gas flows in porous media. Nonlinearity, 31(8):3617-3650, 2018.

[10] Emine Celik, Luan Hoang, and Thinh Kieu. Generalized Forchheimer flows of isentropic gases. J. Math. Fluid Mech., 20(1):83-115, 2018.

[11] J. Chadam and Y. Qin. Spatial decay estimates for flow in a porous medium. SIAM J. Math. Anal., 28(4):808-830, 1997.

[12] L. P. Dake. Fundamentals of reservoir engineering, volume 8 of Developments in Petroleum Science. Elsevier Science B.V., 1978.

[13] Klaus Deimling. Nonlinear functional analysis. Dover Publications, Inc., 2010. Originally published in 1985 by Springer-Verlag.

[14] Emmanuele DiBenedetto. Degenerate parabolic equations. Universitext. Springer-Verlag, New York, 1993.

[15] P Forchheimer. Wasserbewegung durch Boden. Zeit. Ver. Deut. Ing., 45:1781-1788, 1901.

[16] Ph. Forchheimer. Hydraulik. Number Leipzig, Berlin, B. G. Teubner. 1930. 3rd edition.

[17] Avner Friedman. Partial differential equations of parabolic type. Dover Publications, Inc., 2008. Originally published in 1964 by Prentice-Hall, Inc.

[18] Karol W. Hajduk and James C. Robinson. Energy equality for the 3D critical convective Brinkman-Forchheimer equations. J. Differential Equations, 263(11):7141-7161, 2017.

[19] L. Hoang, A. Ibragimov, T. Kieu, and Z. Sobol. Stability of solutions to generalized Forchheimer equations of any degree. J. Math. Sci. (N.Y.), 210(4, Problems in mathematical analysis. No. 81 (Russian)):476-544, 2015. 
[20] Luan Hoang and Akif Ibragimov. Structural stability of generalized Forchheimer equations for compressible fluids in porous media. Nonlinearity, 24(1):1-41, 2011.

[21] Luan Hoang and Akif Ibragimov. Qualitative study of generalized Forchheimer flows with the flux boundary condition. Adv. Differential Equations, 17(5-6):511-556, 2012.

[22] Luan Hoang and Thinh Kieu. Interior estimates for generalized Forchheimer flows of slightly compressible fluids. Adv. Nonlinear Stud., 17(4):739-767, 2017.

[23] Luan Hoang and Thinh Kieu. Global estimates for generalized Forchheimer flows of slightly compressible fluids. J. Anal. Math., 137(1):1-55, 2019.

[24] Luan T. Hoang, Thinh T. Kieu, and Tuoc V. Phan. Properties of generalized Forchheimer flows in porous media. J. Math. Sci., 202(2):259-332, 2014.

[25] David Jerison and Carlos E. Kenig. The inhomogeneous Dirichlet problem in Lipschitz domains. J. Funct. Anal., 130(1):161-219, 1995.

[26] O. A. Ladyženskaja, V. A. Solonnikov, and N. N. Ural'ceva. Linear and quasilinear equations of parabolic type. Translated from the Russian by S. Smith. Translations of Mathematical Monographs, Vol. 23. American Mathematical Society, Providence, R.I., 1968.

[27] Peter A. Markowich, Edriss S. Titi, and Saber Trabelsi. Continuous data assimilation for the three-dimensional Brinkman-Forchheimer-extended Darcy model. Nonlinearity, 29(4):1292-1328, 2016.

[28] Morris Muskat. The flow of homogeneous fluids through porous media. McGraw-Hill Book Company, inc., 1937.

[29] Donald A. Nield and Adrian Bejan. Convection in porous media. Springer-Verlag, New York, fourth edition, 2013.

[30] L. E. Payne, J. C. Song, and B. Straughan. Continuous dependence and convergence results for Brinkman and Forchheimer models with variable viscosity. R. Soc. Lond. Proc. Ser. A Math. Phys. Eng. Sci., 455(1986):2173-2190, 1999.

[31] L. E. Payne and B. Straughan. Convergence and continuous dependence for the Brinkman-Forchheimer equations. Stud. Appl. Math., 102(4):419-439, 1999.

[32] Brian Straughan. Stability and wave motion in porous media, volume 165 of Applied Mathematical Sciences. Springer, New York, 2008.

[33] Peter Vadasz. Fluid flow and heat transfer in rotating porous media. Springer, 2016.

[34] J. C. Ward. Turbulent flow in porous media. Journal of the Hydraulics Division, Proc. Am. Soc. Civ. Eng., 90(HY5):1-12, 1964. 